This is the accepted version of the following article:

Cuxart M.G., Šics I., Goñi A.R., Pach E., Sauthier G., Paradinas M., Foerster M., Aballe L., Fernandez H.M., Carlino V., Pellegrin E.. Inductively coupled remote

plasma-enhanced chemical vapor deposition (rPE-CVD) as a versatile route for the deposition of graphene micro- and nanostructures. Carbon, (2017). 117. : 331 - . 10.1016/j.carbon.2017.02.067,

which has been published in final form at

https://dx. doi.org/10.1016/j.carbon.2017.02.067

https://dx.doi.org/10.1016/j. carbon.2017.02.067. This manuscript version is made 

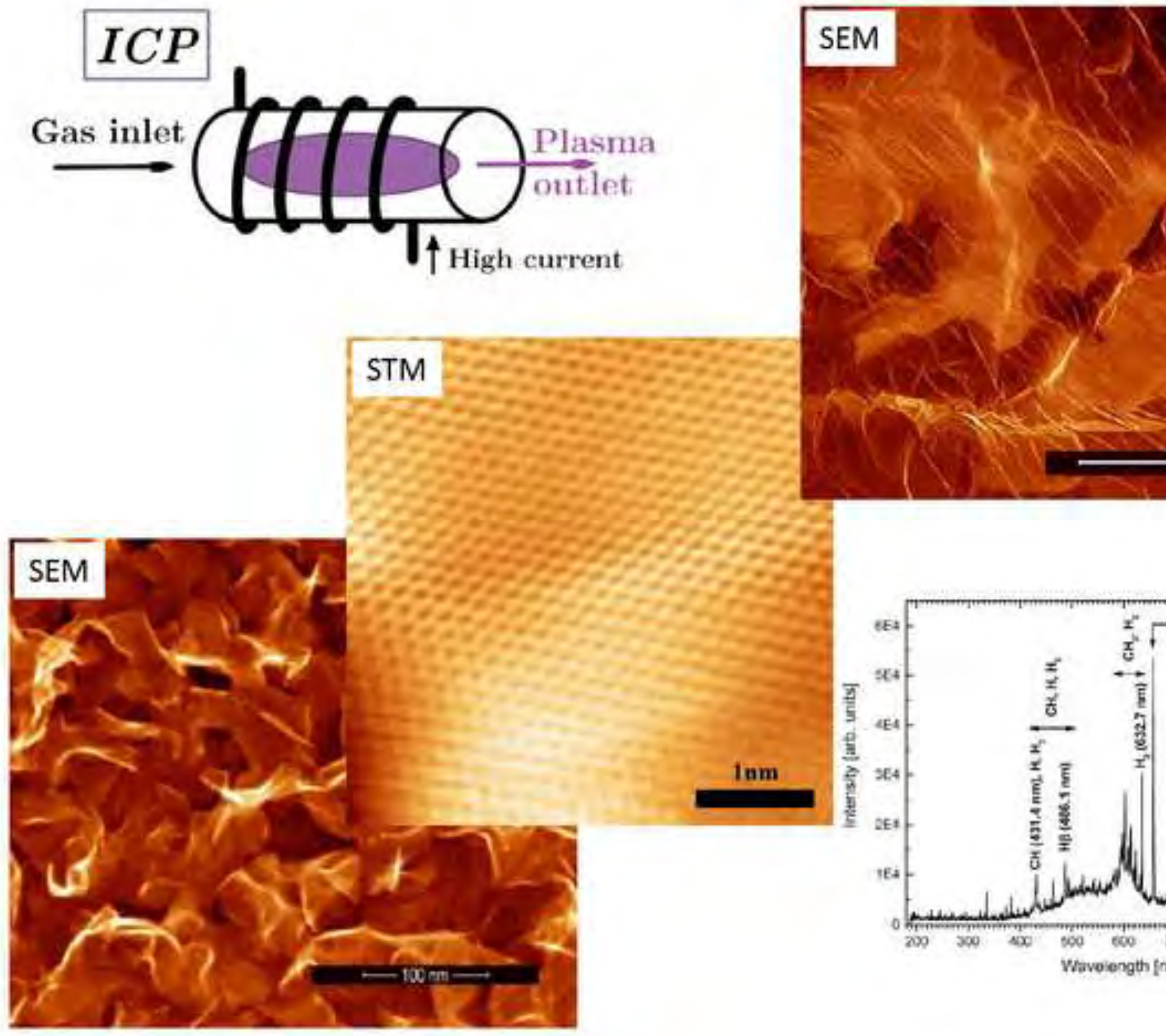


\title{
Inductively Coupled Remote Plasma-Enhanced Chemical Vapor Deposition as a Versatile Route for the Deposition of Graphene Micro- and Nanostructures.
}

\author{
M. G. Cuxart ${ }^{a 1}$, I. Šics ${ }^{a}$, A. R. Goñi ${ }^{b, c}$, E. Pach ${ }^{d}$, G. Sauthier ${ }^{d}$, M. \\ Paradinas $^{d}$, M. Foerster ${ }^{a}$, L. Aballe ${ }^{a}$, H. Moreno Fernandez ${ }^{a}$, V. Carlino ${ }^{e}$, \\ E. Pellegrin ${ }^{a, * 2}$ \\ a ALBA Synchrotron Light Facility, Carrer de la Llum 2-26, 08290 Cerdanyola del Vallès, \\ Spain. \\ ${ }^{b}$ Institut de Ciencia de Materials de Barcelona (ICMAB-CSIC), Campus UAB, Bellaterra, \\ Spain. \\ c ICREA, Passeig Lluis Companys 23, 08010 Barcelona, Spain. \\ ${ }^{d}$ Catalan Institute of Nanoscience and Nanotechnology (ICN2), CSIC and The Barcelona \\ Institute of Science and Technology, Campus UAB, Bellaterra, 08193 Barcelona, Spain \\ e ibss Group Inc., Burlingame, CA 94010, USA.
}

\begin{abstract}
Multiple layers of graphene thin films with micro-crystalline orientation and vertical graphene nano-sheets were grown on different substrates (i.e., polycrystalline nickel foil, $\mathrm{Ni}(111)$, highly oriented pyrolytic graphite) using a singlestep process based on low-pressure remote Plasma-Enhanced Chemical Vapor Deposition (rPE-CVD). In contrast to previous studies, a novel basic approach to this technique including a new remote inductively coupled RF plasma source has been used to (i) minimize the orientational effect of the plasma electrical fields during the catalyst-free growth of graphene nano-sheets, (ii) warrant for a low graphene defect density via low plasma kinetics, (iii) decouple the dissociation process of the gas from the growth pro-

\footnotetext{
1 marc.gonzalez@icn2.cat. Now at the Catalan Institute of Nanoscience and Nanotechnology (ICN2), CSIC and The Barcelona Institute of Science and Technology, Campus UAB, Bellaterra, 08193 Barcelona, Spain

$2{ }^{*}$ Corresponding author. Tel.: +34 93592 4418. E-mail: epellegrin@cells.es
} 
cess of graphene on the substrate, (iv) tune the feedstock gas chemistry in view of improving the graphene growth, and (v) reduce the growth temperature as compared to conventional chemical vapor deposition (CVD). In order to study the various aspects of the rPE-CVD graphene growth modes and to assess the characteristics of the resulting graphene layers Raman spectroscopy, XPS, SEM, and STM were used. The results give evidence for the successful performance of this new rPE-CVD plasma deposition source, that can be combined with in situ UHV-based processeses for the production of, e.g, hybrid metal ferromagnet/graphene systems.

\section{Introduction}

The electrical and mechanical properties of graphene, in particular its ballistic transport properties, have opened up exciting possibilities for this material as a replacement for silicon [1, 2]. Graphene has a simple structure, consisting

5 of a honeycomb arrangement of carbon atoms in a monoatomic layer (with a lattice parameter $a_{g r}=2.46 \AA$ ), and is mechanically and chemically stable. Mechanical exfoliation of highly oriented pyrolytic graphite ( $H O P G$ ) has been the most common method for producing single layers of this material. However, the lateral dimensions of such monolayer samples are typically limited to the microscale. Since large area of defect-free graphene films on insulating substrates are required for practical applications, several techniques have been explored such as the reduction of oxidized graphite films, graphitization of $\mathrm{SiC}$ wafers under ultra-high vacuum chemical vapor deposition $(C V D)$ on transition metals etc.

Recently, several authors [1, 2, 3, 4, 5, 6, 7] have reported new graphene deposition methods using inductively-coupled RF plasma (ICP) as one way to decouple the gas dissociation process from the graphene substrate growth process within the context of Plasma Enhanced Chemical Vapor Deposition (PECVD). This makes this method more tunable as compared to classical CVD as it allows for an independent control of precursor gas dissociation and growth

20 parameters, which should lead to a better control over the size and shape of 
the resulting carbon nanostructures. Moreover, using the precursor gas in plasma state involves, by definition, an enhanced density of chemically active carbon radicals, thus enhancing the deposition rate especially in the case of non-equilibrium low-temperature plasma such as the above ICP RF plasma, including a weakly ionized environment with a relatively high electron energy. By using a highly reactive precursor such as a hydrocarbon gas, one can thus decrease the growth/deposition time and/or the substrate temperature. This latter feature opens the graphene deposition process to a wider variety of substrates with low melting points avoiding, e.g., surface roughening as well as the temperature-induced diffusion of carbon atoms into the substrate, resulting in the subsequent carbon surface segregation after sample cooldown. In addition, further intrinsic CVD limitations like, e.g., the single monolayer growth limit of graphene onto $\mathrm{Ni}(111)$ can easily be defeated by overcoming the passivation of the graphene monolayer on $\mathrm{Ni}(111)$, thus increasing the flexibility of PE-CVD 35 processes further.

Regarding the growth of planar graphene, Boyd et al. 3 have proposed a simple single-step direct PE-CVD deposition method using a quartz tube within an Emerson cavity, encompassing high gas partial pressures of 0.3 mbar (i.e., $0.4 \% \mathrm{CH}_{4}, 0.4 \% \mathrm{~N}_{2}, \mathrm{H}_{2}$ balance) together with a direct plasma and electric 40 field exposure of the $\mathrm{Cu}$ foil, $\mathrm{Cu}(100)$, and $\mathrm{Cu}(111)$ sample substrates, with the good quality graphene therefore being limited to the substrate side not facing the plasma. Nandamuri et al. 4, were using remote PE-CVD on $\mathrm{Ni}$ foil and $\mathrm{Ni}(111)$, with an ICP plasma at relatively high partial gas pressures of 0.12 mbar (i.e., $23.8 \% \mathrm{CH}_{4}, \mathrm{H}_{2}$ balance) in a non-UHV compatible quartz tube reactor setup. On a similar technological basis, Zhang et al. 6, 7] were deposing at similar pressures of 0.266 mbar (i.e., pure $\mathrm{CH}_{4}$ ) on wide range of substrates with rather long deposition times of up to 3 hours for a graphene coverage of 2-3 monolayers resulting into $100 \mathrm{~nm}$ graphene patches on HOPG and nano-crystalline entities on $\mathrm{SiO}_{2}$. Regarding the catalyst-free growth of vertical graphene nano-sheets on $\mathrm{Si}$, Zhu et al. 8 did use PE-CVD with a planar ICP source and a methane (40\%)/hydrogen (60\%) feedstock gas mixture, 
concluding that the correct combination of hydrogen atomic plasma species together with the interaction of the plasma electric field with the growth process results into the growth of vertical graphene nano-sheets.

During the last years, CELLS-ALBA [9] together with ibss Group Inc. [10] have acquired a large expertise regarding the generation and handling of remote (or downstream) low-pressure ICP RF plasma for the cleaning of carbon contaminations as generated by the interaction of the synchrotron photon beam with residual $\mathrm{CO}_{2}$ gas molecules etc. at the surface of synchrotron optics, as well as by the electron beam on samples within SEM or TEM setups [11, 12. An inductively coupled GV10x RF plasma source (ibss Group) has so far been used as a cleaning tool for carbon-contaminated optical surfaces, where the feedstock gas of the plasma consists of a chemically active agent (such as, e.g., oxygen or hydrogen neutral radicals), thus converting the carbon into $\mathrm{CO}_{2}, \mathrm{CO}$, or hydrocarbons gas via oxidation or reduction, respectively. In order to preserve the advantages of the above graphene CVD approaches while striving for an improvement of the graphene deposition process towards an enhanced flexibilty of the graphene growth modes, a better UHV compatibility of the overall process, and an improved graphene sample quality, we have further developed the rPE-CVD technique using the GV10x plasma source but inverting its operation principle (see Figure 1). From a topological perspective we propose a remote ICP plasma generated within a distant/separate plasma tube volume, thus avoiding preferential perpendicular growth directions that electrical fields within a direct plasma deposition process unavoidably incur. Furthermore, a direct plasma should facilitate a deposition of carbon nano-structures with significantly reduced defect density. The unsurpassed chemical flexibility and tunability of PE-CVD processes supporting the simultaneous reaction of different chemical species makes up a major advantage as compared to traditional so CVD processes. 13, 14. Last but not least, the overall vacuum characteristics of the employed GV10x source result into a deposition process that is compatible with generic and thus unavoidable UHV requirements of prior or 
subsequent MBE processes, in view of allowing for, e.g., the production of ferromagnet/graphene/ferromagnet hybrid multilayer thin film sample systems.

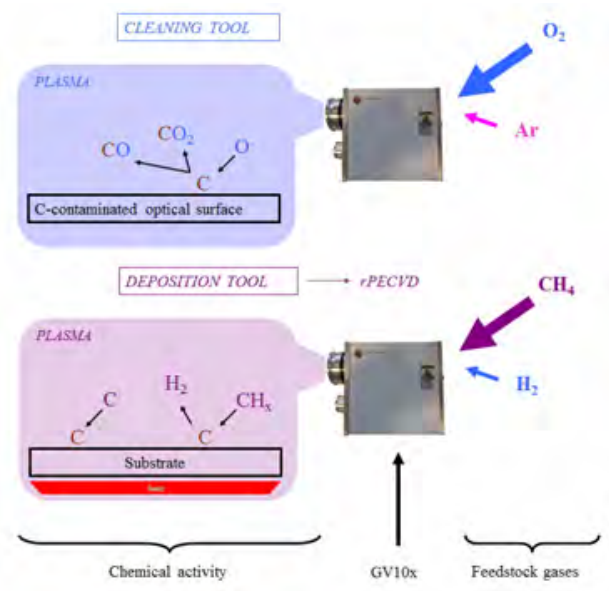

Figure 1: Reversing the GV10x plasma source from a top-down plasma cleaning tool to a bottom-up deposition tool for remote plasma-enhanced chemical vapor deposition (rPE-CVD).

In order to accomplish the purpose of inverting the working principle of the GV10x from a top-down cleaning tool to a bottom-up deposition tool, several routes for depositing graphene onto different substrates were explored over a wide range of deposition parameters. The substrates studied were $\mathrm{Ni}$ foil, HOPG(0001) and $\mathrm{Ni}(111)$ (Note: A complete set of experimental data for the case of the early graphene growth stages on $\mathrm{Ni}(111)$ can be found in the Supplementary Content). The resulting samples were characterized following a systematic approach in order to verify the deposited graphene layer thickness, morphology, defect density etc. Raman spectroscopy (using a laser with $632.8 \mathrm{~nm}$ wavelength) gives a precise idea of the kind of carbon allotrope and crystalline quality [15]. Scanning Electron Microscopy (SEM) and Scanning Tunnelling Microscopy (STM) yield a direct picture of the sample surface with a nanometric and atomic resolution, respectively. Finally, X-Ray Photoemission Spectroscopy (XPS) gives a detailed chemical analysis of the surface that allows calculating atomic concentrations and thickness in terms of deposited monolayers. The main point of this characterization procedure is to 
cross-check results and to get a more complete picture of the characteristics of the produced rPE-CVD graphene samples, the pertinent growth processes and dynamics as a function of sample substrate and process parameters (e.g., RF power, feedstock gas mixtures, total pressure, susbtrate temperature etc.).

105

\section{Experimental}

There are several approaches for creating a plasma using RF electromagnetic (EM) fields. In high pressure plasma, the energy that the electrons have acquired from the EM fields is more likely to be transferred to the heavy ions, because of the higher collision probability. This results into a thermalisation of the whole gas, so that $T_{e} \simeq T_{i}=T_{\text {gas }}$. Plasma fulfilling this condition are called thermal plasma working close to atmospheric pressure. On the other hand, plasmas are termed non-thermal when $T_{e} \gg T_{i}=T_{g a s}$, and they work in lower pressure ranges $\left(P_{a t m}-10^{-3}\right.$ mbar $)$. Thermal plasma are usually more powerful, while non-thermal plasma are more selective and are thus typically used for produces non-thermal plasma through inductively coupled RF discharges (see below).

There are two well-known methods for the generation of the non-thermal plasma created by RF discharges:

(i) Capacitively Coupled Plasma $(C C P)$ : $\mathrm{CCP}$ discharges provide the EM field to the gas flow by means of electrodes located either outside or inside of the plasma chamber (see Figure 2). These discharges primarily stimulate a DC or AC electric field in order to facilitate plasma ignition. High AC or DC electric fields are needed for this purpose.

125

(ii) Inductively Coupled Plasma (ICP): An in-air coil surrounding a ceramic tube inside which the EM fields are induced into the gas flow (see Figure 22. A high RF electric current passes through the coil which induces a high frequency magnetic field along the tube axis. This AC magnetic field induces a high frequency vortex electric field, concentric with the 
elements of the coil and providing the plasma breakdown and sustaining the plasma. Non-thermal ICP plasma discharges work at low pressures (i.e., $10^{-1}$ to $10^{-3}$ mbar in our setup), so that the ratio between electric field and pressure $(E / p)$ is sufficient for ionization. The coupling between plasma and the coil can be seen as a voltage decreasing transformer, where the coil works as the primary windings and the plasma as the secondary windings. ICP discharges are operated at high currents, high electron concentration, and high electrical conductivity.

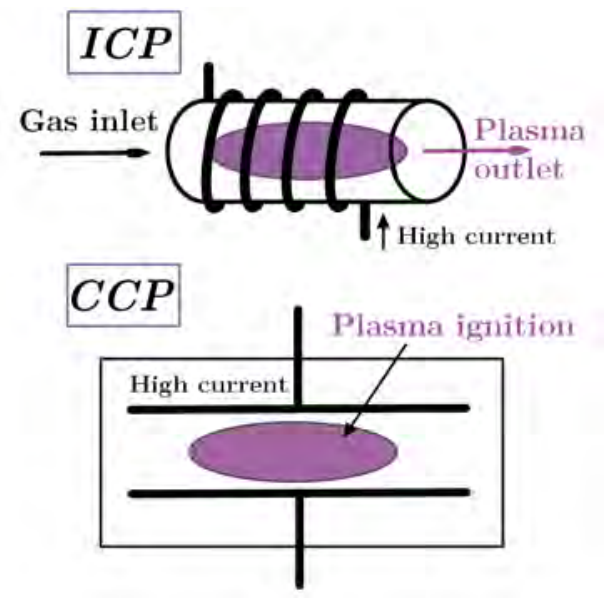

Figure 2: Schematic of the Capacitively Coupled Plasma $(C C P)$ and Inductively Coupled Plasma $(I C P)$ sources.

From the perspective of thin film deposition, the CCP setup includes the installation of the sample substrate onto one of the capacitor antenna plates (i.e., either connected to the RF source or to ground), thus exposing the sample directly to the plasma and the associated electric fields together with highenergy ions and electrons. While this may be desirable for ion-assisted plasma processes, the growth of graphene thin films with low defect densities will unavoidably suffer from this [3]. In addition, sputtering products from the antenna material or from other chamber parts (especially from the build-up of a DC bias on floating/insulating parts inside the plasma chamber) may possibly lead to a corresponding contamination of the thin film and/or the substrate. 
On the other hand, Remote ICP plasma sources offer the advantage of not exposing the RF coil or antenna material to the plasma (i.e., avoiding a source of plasma and/or thin film contaminations). In addition, the inherent geometrical separation of the plasma generation volume from the thin film deposition chamber volume keeps the latter free of the EM fields as generated by the upstream RF coil. Thus, the overall topology warrants for the low kinetic energies of ions and electrons within the downstream deposition chamber.

Last but no least, it should be pointed out that the high currents and high electron densities as generated in an ICP plasma sources as compared to CCP setups is beneficiary for the efficient production of atomic hydrogen, 13 thus facilitating the defect-free growth of graphene nano-structures at lower hydrogen gas concentrations. This will become apparent from the low hydrogen feedstock gas concentrations used in this study as compared to, e.g., the results from other

PE-CVD graphene deposition studies mentioned above.

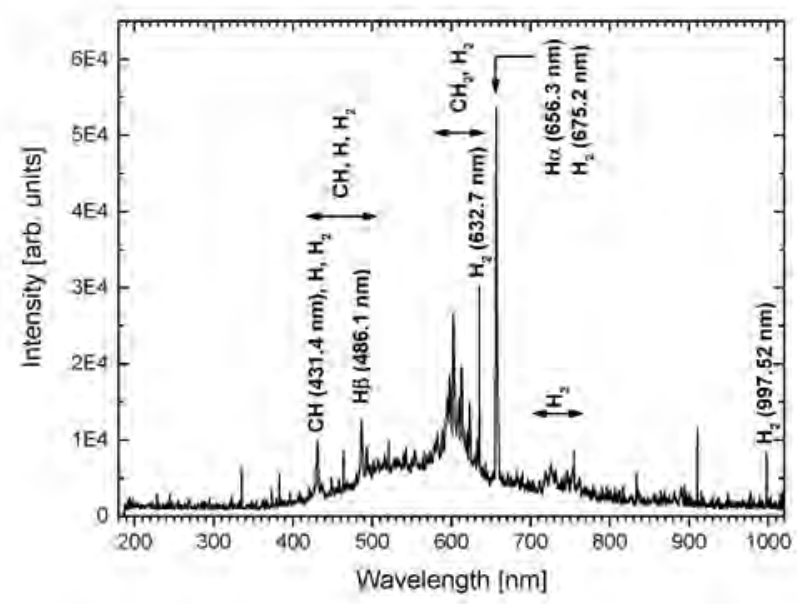

Figure 3: Optical emission spectrum (OES) from the $\mathrm{CH}_{4} / \mathrm{H}_{2} 80 \% / 20 \%$ feedstock gas plasma used for the growth of the Ni foil sample 3 .

Figure 3 shows a typical optical emission spectrum (OES) from a $\mathrm{CH}_{4} / \mathrm{H}_{2}$ feedstock gas plasma. From this, the atomic type, charge, as well as the precise 
mixing ratio of the chemically active species within the plasma can be determined, thereby enabling a correlation between the plasma properties and the characteristics of the resulting thin film samples. The evaluation performed using commercial software [16] based on tabulated standard emission spectra.[17] The plasma parameters for this OES were those used for the rPE-CVD growth of graphene of the Ni foil sample 3 (see Table 1 ).

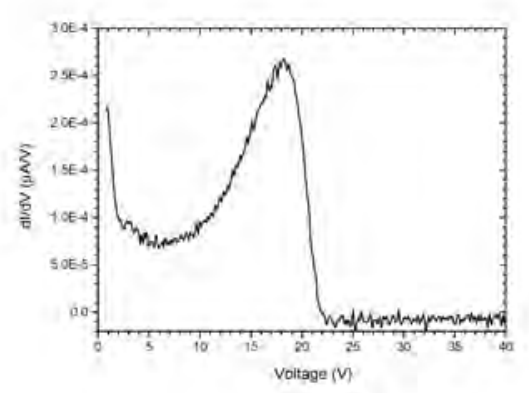

(a) Ion energy distribution function (IEDF) for Ar cations.

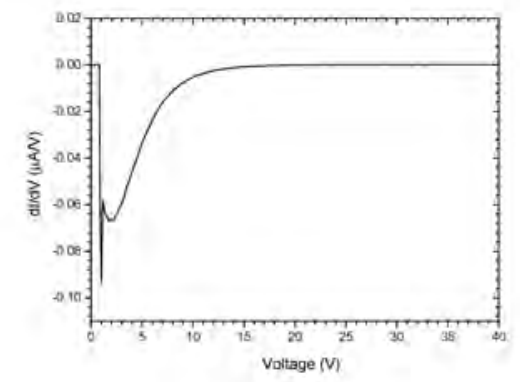

(b) Electron energy distribution function (EEDF).

Figure 4: Typical dI/dV curves based on I-V Faraday cup data taken from a pure Ar feedstock gas plasma (70 W RF power, 0.005 mbar Ar feedstock gas pressure) yielding the Ar cation and electron energy distribution functions at $800 \mathrm{~mm}$ from the plasma source.

As a further example for the characteristics of the downstream (or afterglow) plasma as generated by the GV10x plasma source during the rPE-CVD deposition process, Figure 4 a shows typical dI/dV curves as obtained from Fara- 
day cup measurements from a pure Ar plasma ( $70 \mathrm{~W}$ RF power, 0.005 mbar Ar feedstock gas pressure). As the dI/dV curve in Fig. 4a represents the ion energy distribution function (IEDF) for Ar cations, their distinctly low kinetic energies with an average kinetic energy of $13.3 \mathrm{eV}$ could be determined. On the other hand, the electron energy distribution function (EEDF) in Fig. $4 b$ results in an average electron kinetic energy of $4.1 \mathrm{eV}$. In analogy to these data from a pure Ar plasma, we tentatively expect correspondingly low-energy IEDF and EEDF also for the case of a $\mathrm{CH}_{4} / \mathrm{H}_{2} 80 \% / 20 \%$ feedstock gas plasma. As will be shown later on, this warrants for the growth of graphene materials with low defect densities for rPE-CVD deposition in contrast to standard PE-CVD processes based on direct plasma deposition in conjunction with high electric fields and correspondingly high kinetic energies of the charged species within the plasma.

In the next section, we will show how the above technical aspects of an ICP plasma source within the context of a UHV-based rPE-CVD sample preparation chamber setup combine into the efficient deposition of highquality micro-crystalline graphene layers and vertical graphene nanosheets.

\section{Results and Discussion}

The main techniques used to characterize our samples were Raman spectroscopy and SEM. Room temperature STM measurements were carried out for selected samples in order to asses the quality of the graphene foils at the atomic level (i.e., the density of defects as vacancies, antisites, dislocations, boundaries, etc.). Raman spectra of our samples typically show the three characteristic peaks denoted $D\left(1345 \mathrm{~cm}^{-1}\right), G\left(1583 \mathrm{~cm}^{-1}\right)$ and $2 D\left(\sim 2690 \mathrm{~cm}^{-1}\right)$ [18, 15, 19].

According to common knowledge, the following features turn out to be the best fingerprints for graphene (see Fig. 1 in the Supplementary Content document): 
(i) The position of the G peak should be up-shifted by $3-5 \mathrm{~cm}^{-1}$ with respect to that of bulk graphite. 20.

(ii) The 2D peak in bulk HOPG consists of two components, with roughly $1 / 4$ short deposition times as compared to some CVD processes.

It is well-known that atomic hydrogen plays an important role in carbon thin film growth by removing amorphous and $s p^{3}$ hybridized carbon faster than $s p^{2}$ hybridized carbon [8. Hence, the amount of hydrogen radicals (i.e., as ${ }_{230}$ compared to pure $\mathrm{CH}_{4}$ feedstock gas) was increased by introducing additional $\mathrm{H}_{2}$ gas into the feedstock gas mixture. 
Table 1: Summary of experimental conditions for each sample reported in this work (Note: Data corresponding to graphene samples synthesized on $\mathrm{Ni}(111)$ are shown in the Supplementary Content).

\begin{tabular}{|c|c|c|c|c|c|}
\hline $\begin{array}{l}\text { Sample no., } \\
\text { substrate, and result }\end{array}$ & $\begin{array}{l}\text { Feedstock } \\
\text { gases }\end{array}$ & $\begin{array}{l}\text { RF } \\
\text { Power } \\
{[\mathbf{W}]}\end{array}$ & Pressure & Temp. & $\begin{array}{l}\text { Exp. } \\
\text { Time } \\
{[\mathrm{min}]} \\
\end{array}$ \\
\hline $1 \mathrm{Ni}$ foil, partial coating & $\mathrm{CH}_{4} / \mathrm{H}_{2}$ & 207 & $1.0 \cdot 10^{-1}$ & 715 & 1 \\
\hline $2 \mathrm{Ni}$ foil, shrink wrap & $\mathrm{CH}_{4} / \mathrm{H}_{2}$ & 191 & $1.0 \cdot 10^{-1}$ & 725 & 3 \\
\hline $3 \mathrm{Ni}$ foil, cont. shrink wrap & $\mathrm{CH}_{4} / \mathrm{H}_{2}$ & 207 & $1.0 \cdot 10^{-1}$ & 725 & 5 \\
\hline $4 \mathrm{Ni}$ foil, thick disordered & $\mathrm{CH}_{4} / \mathrm{H}_{2}$ & 201 & $1.0 \cdot 10^{-2}$ & 720 & 5 \\
\hline $5 \mathrm{Ni}$ foil, nucleation & $\mathrm{CH}_{4} / \mathrm{H}_{2}$ & 99 & $1.0 \cdot 10^{-1}$ & 625 & 5 \\
\hline $6 \mathrm{Ni}$ foil, granular growth & $\mathrm{CH}_{4}$ & 207 & $1.0 \cdot 10^{-1}$ & 740 & 5 \\
\hline $1 \mathrm{Ni}(111)$, partial coating & $\mathrm{CH}_{4} / \mathrm{H}_{2}$ & 180 & $1.0 \cdot 10^{-1}$ & 725 & 5 \\
\hline $2 \mathrm{Ni}(111)$, nucleation & $\mathrm{CH}_{4} / \mathrm{H}_{2}$ & 189 & $1.0 \cdot 10^{-1}$ & 625 & 5 \\
\hline 1 HOPG(0001), 2D growth & $\mathrm{CH}_{4} / \mathrm{H}_{2}$ & 99 & $2.0 \cdot 10^{-1}$ & 600 & 180 \\
\hline 2 HOPG(0001), nano-sheets & $\mathrm{CH}_{4} / \mathrm{H}_{2}$ & 99 & $1.0 \cdot 10^{-1}$ & 600 & 30 \\
\hline
\end{tabular}

All the typical graphene features in the Raman spectra described above can be observed with different weights for each individual sample as shown in Figure 5 and analyzed in Table 2 Sample 1 presents a Raman spectrum with a very ${ }_{235}$ low $2 \mathrm{D} / \mathrm{G}$ ratio and a low $\mathrm{G} / \mathrm{D}$ ratio (see Table 2), which suggests that carbon has been deposited in a very disordered manner, leading to a large number of defects such as patch edges together with low number of graphene monolayers. This is corroborated by the SEM images in Figure 6a, that show inhomogeneously as well as homogeneously coated areas, where we tentatively ascribe the bright spotted areas in the former to domains with an enhanced defect concentration including a partial coating, with both phenomena contributing to the $\mathrm{D}$ peak. As can be seen from the PEEM/LEEM results in the Supplementary Content, these micron-size bright/spotted domains during the further growth process evolve into carbon-coated non-crystalline domains, in contrast 


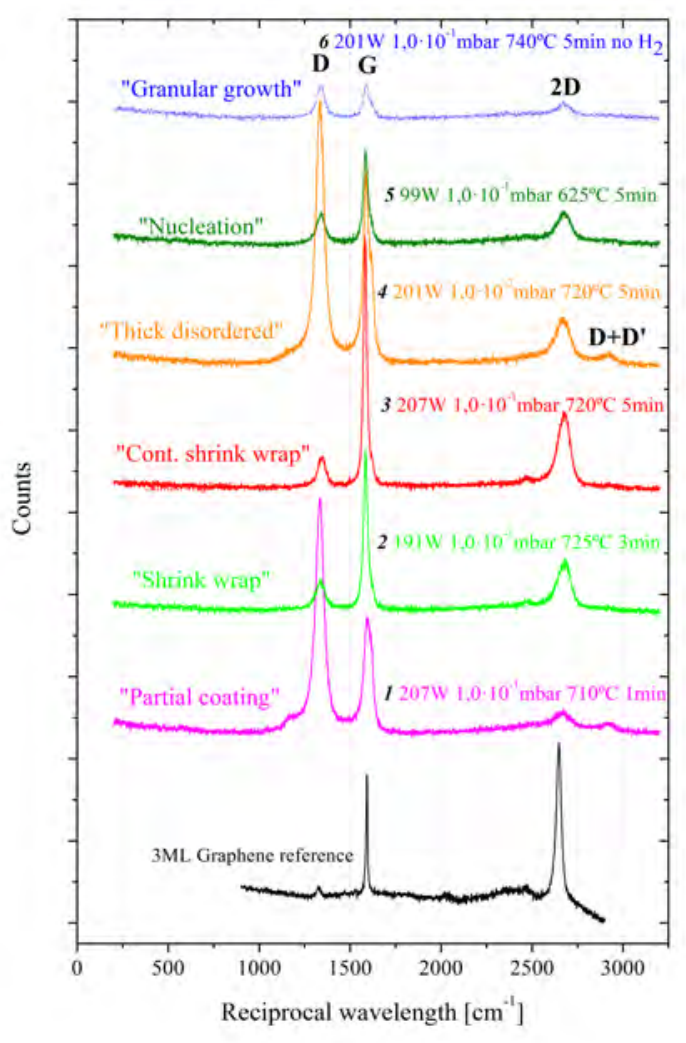

Figure 5: Raman spectra of PECVD graphene samples 1 to 6 grown on Ni foil substrates, together with a 3 ML-graphene reference sample.

to the dark graphene domains exhibiting crystalline characteristics. These darker domains in Figure 6a yield a distinct homogeneous graphene coverage (see also Figure $6 \mathrm{~b}$ for more detail) in form of a wrinkled semitransparent foil. As in the case of CVD graphene on cold-rolled copper foil 22], where the micro-crystalline grains within the $\mathrm{Cu}$ foil surface are known to predominantly reorient with a (100) surface termination at the elevated temperatures used during CVD growth of graphene, we conclude that areas with different amounts of graphene coverage - coinciding with the domains of the $\mathrm{Ni}$ micro-crystals - depend on the orientation of the micro-crystals within the $\mathrm{Ni}$ 255 foil surface, thus influencing the graphene growth rate. Everything considered, 
Table 2: Qualitative analysis of the graphene features measured in each sample on Ni foil. (a), (b) and (c) refer to the three Raman fingerprints of graphene: Shift of G peak, width of the $2 \mathrm{D}$ peak, and ratio between $2 \mathrm{D}$ and $\mathrm{G}$ peaks. The last column with the ratio between the $\mathrm{G}$ and the $\mathrm{D}$ peaks intensities gives a relative measure of the amount of defects within the graphene layers. The D' peak were taken into account for the intensity evaluation of the G peak.

\begin{tabular}{lllll}
\hline $\begin{array}{lll}\text { Sample no., } \\
\text { substrate, and result }\end{array}$ & $(\boldsymbol{a})$ & $(\boldsymbol{b})$ & $(\boldsymbol{c})$ & $(\boldsymbol{d})$ \\
& $\begin{array}{l}\boldsymbol{p o s}_{G} \text { (meas.) } \\
-\boldsymbol{p o s}_{G}(\mathbf{H O P G})\end{array}$ & $\mathbf{I}_{2 D} / \mathbf{W i d t h}_{2 D}$ & $\mathbf{I}_{2 D} / \mathbf{I}_{G}$ & $\mathbf{I}_{G} / \mathbf{I}_{D}$ \\
& {$\left[\mathrm{~cm}^{-1}\right]$} & {$[\mathrm{cm}]$} & & \\
\hline (1) Ni foil, partial coating & 10.3 & 0.23 & 0.18 & 0.32 \\
(2) Ni foil, shrink wrap & 2.9 & 1.32 & 0.86 & 2.27 \\
(3) Ni foil, cont. shrink wrap & 1.2 & 2.51 & 0.78 & 3.88 \\
(4) Ni foil, thick disordered & 5.2 & 0.92 & 0.46 & 0.44 \\
(5) Ni foil, nucleation & 3.2 & 0.75 & 0.81 & 1.53 \\
(6) Ni foil, granular growth & 8.8 & 1.28 & 0.58 & 0.81 \\
\hline
\end{tabular}

these results speak in favour of increasing the (low) deposition time $(1 \mathrm{~min})$ as used for sample 1. The area with a low graphene coverage in the SEM image for sample 1 (see Fig. 6a), are reminiscent of the early rPE-CVD growth stage of graphene on $\mathrm{Ni}(111)$ discussed in the Supplementary Content (see Fig. $3 c$ in this document). There, we could observe an evolution from "spider"-shaped seed patches at the edges of the $\mathrm{Ni}(111)$ terraces via higher deposition temperature into the larger graphene patches also seen for the Ni foil sample 1 in Fig. 6a.

We thus speculate that the low/slow graphene growth areas do correspond to Ni micro-crystals with (111) surface orientation, while other areas including larger growth rates do represent other $\mathrm{Ni}$ surface orientations. An indepth micro-LEED or low-energy electron microscopy (LEEM) analysis would be needed in order to prove this point.

Samples 2,3 , and 5 show similar Raman spectra, but now with strongly reduced defect concentrations (see Figure 5. Also, samples 2 and 3 exhibit higher 


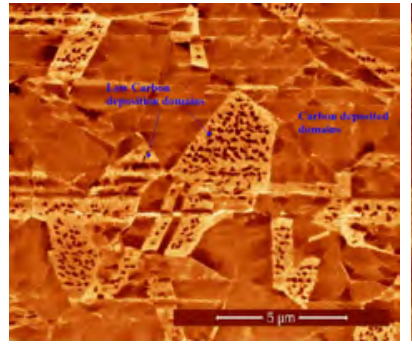

(a) Sample 1

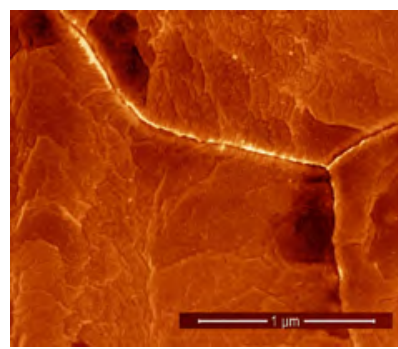

(d) Sample 2

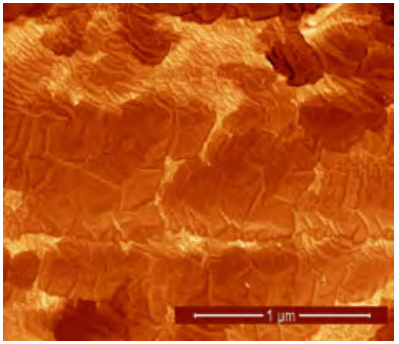

(b) Sample 1

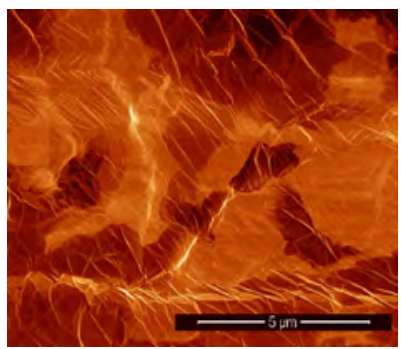

(e) Sample 3

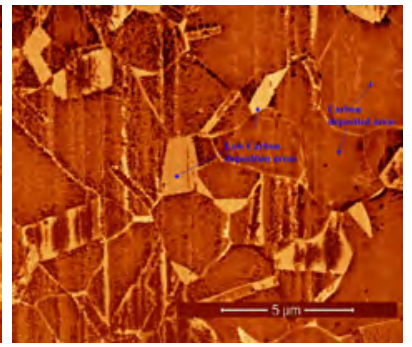

(c) Sample 2

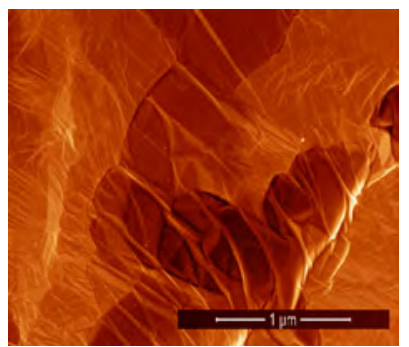

(f) Sample 3

Figure 6: SEM images for sample 1; Sample 2; Sample 3 "Shrink wrap". All of them grown on polycrystalline $\mathrm{Ni}$ foil.

absolute intensities as well as a sharper 2D peak than in sample $\boldsymbol{5}$ (as seen in the $\mathrm{I}_{2 D} / \mathrm{Width}_{2 D}$ ratio in Table 2), which can be explained by the higher synthesis temperature of the former two samples $\left(\sim 725^{\circ} \mathrm{C}\right.$ against $\left.625^{\circ} \mathrm{C}\right)$. This is confirmed in the SEM images: Sample $2(3 \mathrm{~min})$ shows a more extensive and more homogeneous graphene coverage than sample 1 (1 min deposition time; see Figs. 6c and 6d). Sample 3 (3 min) shows a thicker and very homogeneous graphene coverage (referred to as "shrink wrap") and appears to be in a advanced stage of growth. In contrast to this, sample 5 only exhibits a partial "shrink wrap" appearance (Figure $7 \mathrm{c}$ and $7 \mathrm{~d}$ ), and is still mostly transparent with respect to the micro-crystalline domains of the nickel substrate (see the SEM images for the clean nickel reference substrate in the Supplementary Content). The nucleation stage of the graphene growth in this sample 5 can be explained by both the lower substrate temperature and the lower plasma RF power applied during the growth process. We also note that sample 3 appears to be the only sam- 


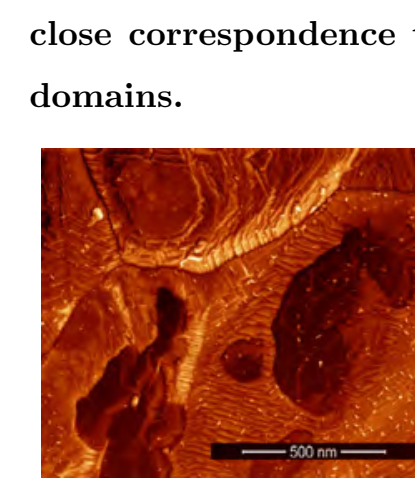

(a) Sample 4

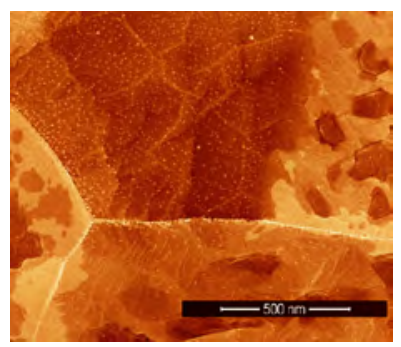

(d) Sample 5

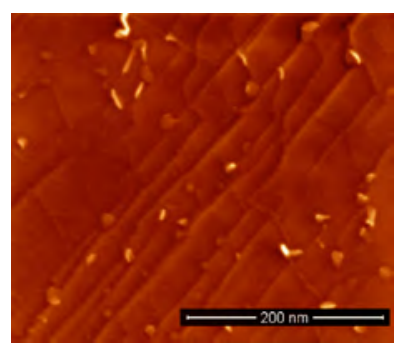

(b) Sample 4

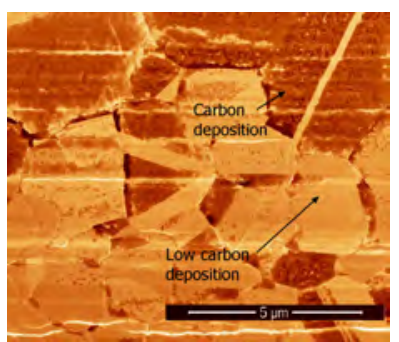

(e) Sample 6

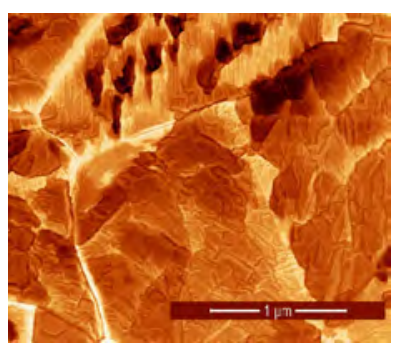

(c) Sample 5

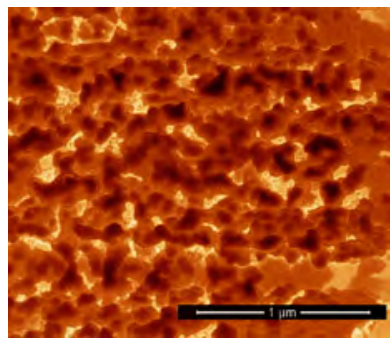

(f) Sample 6

Figure 7: SEM images for sample 4 "Thick disordered layers"; Sample 5 "Nucleation"; Sample 6 "Granular growth". All of them grown on polycrystalline Ni foil.

Samples 1 and 4 present high D peaks: Too short deposition times (1 min for sample 1) or low deposition pressures (0.01 mbar for sample 4) turned out to be non-optimum. A lower plasma feedstock gas pressure implies a larger mean free path length for the chemically active carbon and hydrogen radicals within the plasma (as obvious from a brighter downstream plasma inside the deposition chamber), thus inducing an accelerated, rather three-dimensional graphene 
"step flow" growth process resulting into a thick disordered graphene deposition. The consequences resulting from this accelerated 3D growth mode can be observed from the resulting rather complex and thick disordered stepped carbon terrace structures in Figure $7 \mathrm{a}$ and $7 \mathrm{~b}$, which include a large density of boundaries at the step edges, the deposition of scattered grains of about $20 \mathrm{~nm}$ length (see Figure 7b), and a larger contribution of an amorphous carbon phase that broadens the Raman peaks (see Figure 5). Since the characteristic diamond peak $\left(1332 \mathrm{~cm}^{-1}[23]\right)$ is located very close to the D peak, we speculate that the growth of a $s p^{3}$ phase in sample 4 may also induce the broadening of the $\mathrm{D}$ peak as observed in samples 1 and 4 as compared to, e.g., sample 3 . With the overall surface morphology of sample 4 appearing to be rather complex, the internal chemical/structural aspects of the above stepped terraces could not be investigated with the experimental tools being at hand and are presently not clear.

X-ray photoelectron spectroscopy (XPS) measurements were carried out using a monochromatized $\mathrm{Al} \mathrm{K}_{\alpha} \mathrm{X}$-Ray source. In addition to the SEM images giving evidence for the different $2 \mathrm{D}$ versus $3 \mathrm{D}$ growth modes induced by the lower deposition pressure when going from sample 3 to 4 , the XPS survey spectra shown in Fig. 8 further corroborate the larger graphene layer thickness for the latter via the larger $\mathrm{C} 1 \mathrm{~s} / \mathrm{Ni2p}$ XPS line ratio, which is also reflected by its smaller $\mathrm{I}_{2 D} / \mathrm{I}_{G}$ ratio. Thus, we conclude that a factor of ten in the plasma deposition pressure (while keeping all other parameters constant) results in a significant changeover in the growth characteristics as well as the underlying graphene growth mechanisms, indicating a high dynamical criticality of the graphene growth via rPE-CVD.

As sample 3 exhibited the lowest defect concentration in the Raman spectra (see Figure 5) and the most promising SEM pictures in terms of a complete and homogeneous substrate coverage (see Figures 6e and 6f), an estimation of the number of nanocrystalline graphene layers was carried out using the above XPS data together with the SESSA software (Simulation of Electron Spectra 


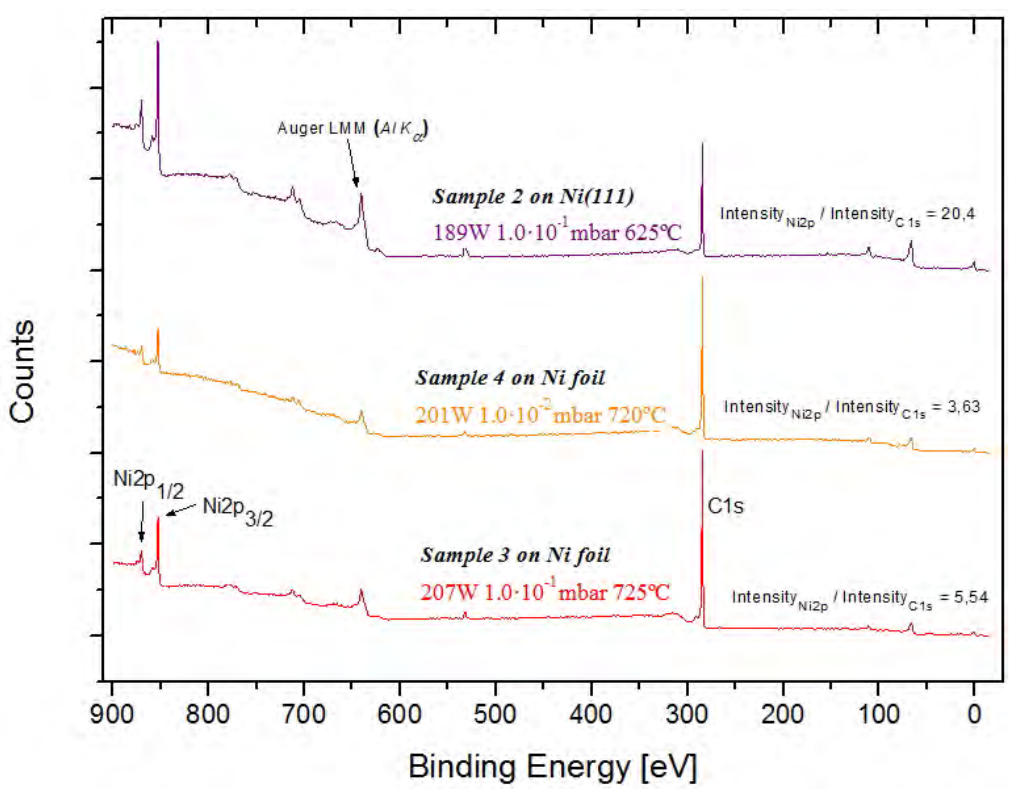

Figure 8: XPS survey spectra of graphene on nickel foil sample 3 (bottom) and 4 (middle), together with the XPS survey spectrum of graphene on Ni(111) sample 2 shown in the Supplementary Content (top).

for Surface Analysis [24]). Simulations were based on a simple model of an infinitely thick nickel substrate covered by a thin carbon layer. XPS spectra were simulated for several carbon thicknesses of $3.35 \AA$ (1 monolayer (1GL)), $6.70 \AA(2 \mathrm{GL}), 10.05 \AA(3 \mathrm{GL}), 13.40 \AA$ (4GL), $16.75 \AA(5 \mathrm{GL}), 20.10 \AA(6 \mathrm{GL})$ and $23.45 \AA$ (7GL) (one carbon/graphene layer (GL) correspoding to $3.35 \AA$ thickness 25]) and compared with the measured XPS overview spectrum.

As can be observed in Figure 9, the $\mathrm{C} 1 \mathrm{~s}$ and Ni2p peak intensities and peak ratios of the $6 \mathrm{ML}$ simulation and the measured XPS overview spectrum match each other quite accurately $\left(\eta_{N i 2 p_{1 / 2}}=29.1 \%, \eta_{N i 2 p_{3 / 2}}=1.1 \%\right.$ and $\left.\eta_{C 1 s}=4.2 \%\right) . \eta_{\text {peak }}$ values are the relative errors between the simulated and measured intensity for each peak.

Based on the observation that sample 3 exhibits a 6 ML graphene coverage while still maintaining the graphene layer quality, we conclude that the rPE-CVD deposition technique obviously easily overrides the intrinsic graphene 


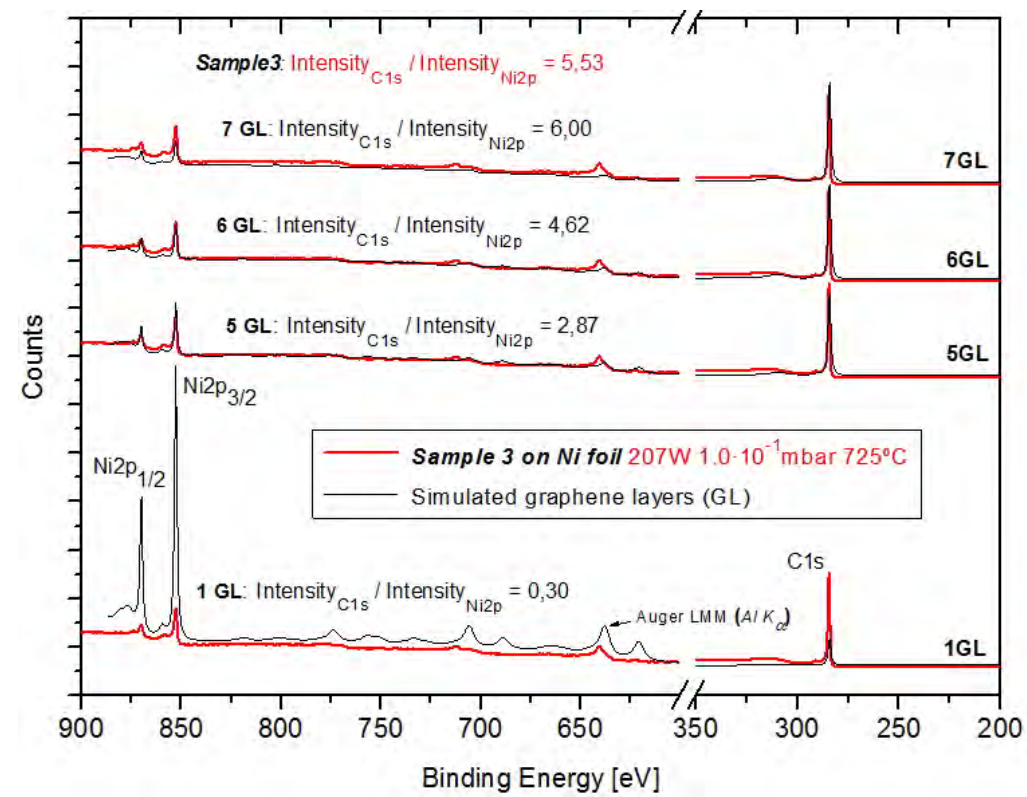

Figure 9: XPS spectrum of graphene on nickel foil 3 (red solid line), together with the simulated XPS spectra calculated with SESSA ranging from one to seven carbon monolayers on a thick nickel substrate (black solid lines).

monolayer limitation on, e.g., $\mathrm{Ni}(111)$ surfaces by overcoming the passivating effect of a graphene monolayer on Ni surfaces (as can be expected from a PE-CVD process).

Atomically-resolved STM images corresponding to sample 2 shown in Fig. 10 were measured on different macroscopic regions that represent distinct topographic features: (i) a flat area where the characteristic honeycomb lattice of the graphitic materials is observed (Fig. 10b); (ii) a wavy area close to a defect of the polycrystalline Ni substrate (Figs. $10 \mathrm{a}$ and $10 \mathrm{c}$, where regions exhibiting different periodic structures: honeycomb, hexagonal and stripped patterns (red, blue, and green dashed squares, respectively), that suggest the presence of regions with different graphene layer stacking sequences [26, 27]. In Fig 10c, local in-plane compressions/expansions of the graphene network are responsible of the smooth transitions through substrate domains, thus quenching the Nickel boundary defects, and maintaining the film quality (see Fig. 10c). More- 
over, monocrystalline flakes of at least 100x100 $\mathrm{nm}^{2}$, exhibiting a defect-freee graphene lattice were measured by STM, which is consistent with the low defect peak measured for this sample by Raman (Figure 5). The characteristics of the film as shown in these STM images for sample 2 should also be typical for sample 3, due to the similar non-linear growth parameters (i.e., RF power and pressure) and SEM appearances.

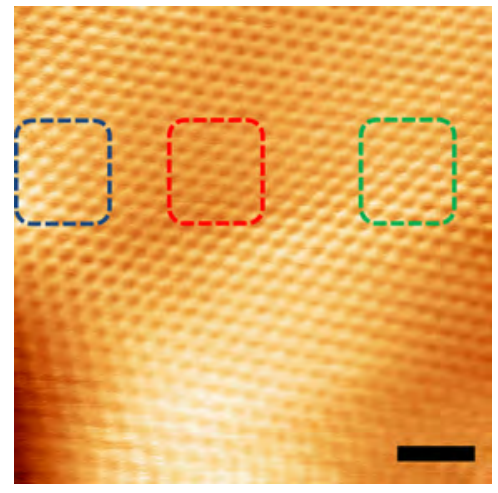

(a) Scale bar $1 \mathrm{~nm}$

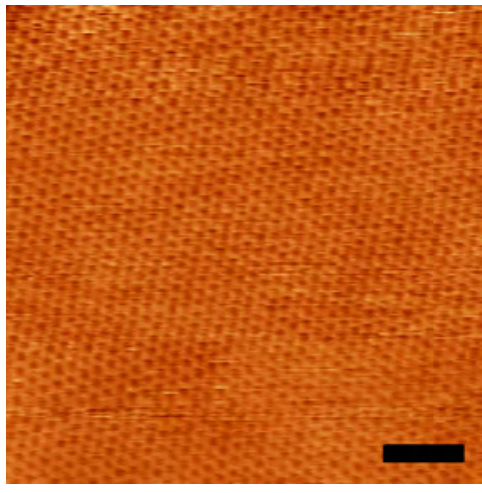

(b) Scale bar $1.4 \mathrm{~nm}$

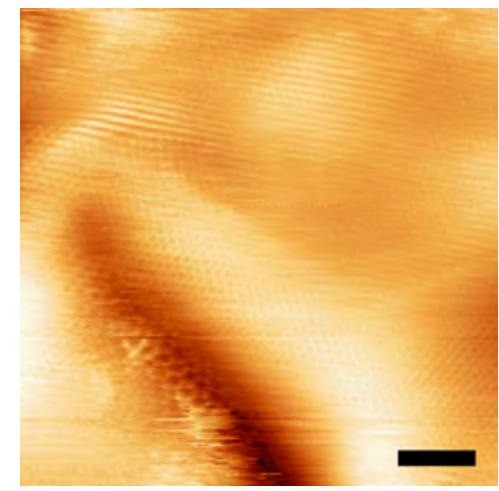

(c) Scale bar $2 \mathrm{~nm}$

Figure 10: High-resolution STM images of sample 2 measured at room temperature.

Topographic images of sample $\mathbf{2}$ were measured at room temperature using a variable-temperature STM (SPECS, STM Aarhus 150) and processed using a freeware software (WSxM 5.0 develop 6.5) [28].

As another important parameter regarding the growth of graphene thin films, 
we have studied the effect of $\mathrm{H}_{2}$ when used as an additional plasma feedstock gas by comparing the Raman spectra of two samples grown under the otherwise identical conditions (i.e., samples 3 and $\boldsymbol{6}$ ), but without $\mathrm{H}_{2}$ in the latter case (see sample 6 , Figure 5). The G/D peak ratio turns out to be much lower, and the $2 \mathrm{D}$ peak appears to be broader for sample 6 (see $\mathrm{I}_{2 D} / \mathrm{Width}_{2 D}$ ratio in Table 22. The SEM images for sample 6 corroborate this, since apart from the graphitic growth mode, there is a three-dimensional growth that comes together with a granular texture (Figure $7 \mathrm{e}$, zoomed in Figure $7 \mathrm{f}$ ). Hence, this confirms that the presence of $\mathrm{H}_{2}$ as an addition to the feedstock gas in the plasma favors the growth rate of $s p^{2}$ phases as compared to other phases like, e.g., $s p^{3}$ and/or (mixed) amorphous phases. According to our previous experience using the GV10x as a plasma source to clean carbon contaminations, this result does not come as a surprise. The cleaning rates of different carbon depositions measured in our experiments using $\mathrm{H}_{2} / \mathrm{Ar}$ plasma revealed that $s p^{3}$ carbon cleans faster $(1.22 \AA / \min )$ than amorphous carbon $(0.59 \AA / \min )$. 12 Since amorphous carbon mostly consists of a mix of $s p^{2}$ and $s p^{3}$, one can conclude that the presence of $s p^{2}$ slows down the reaction during the cleaning process or, in other words, $s p^{3}$-bonded or amorphous carbon is more likely to be converted into hydrocarbons by means of hydrogen radicals during the $\mathrm{H}_{2} / \mathrm{Ar}$ plasma cleaning while $s p^{2}$-bonded carbon is preserved.

An analysis of the C1s XPS spectrum of sample 3 (see Figure 11) reveals the relative concentration of carbon species within the total carbon content (see table insert in Figure 11). As expected, graphitic carbon $\left(s p^{2}\right.$ at $284.5 \mathrm{eV}$ binding energy (BE), forming the 2-dimensional hexagonal lattice) is the most dominant contribution (67.1\%), plus some diamond-like carbon $\left(s p^{3}\right.$ at $285.2 \mathrm{eV}$ $\mathrm{BE}$, forming a diamond structural phase, $12.6 \%$ ), some contribution from oxidized carbon (C-O-C at $285.8 \mathrm{eV}$ BE, $14.5 \%$ ), and the $\pi$ - $\pi^{*}$ shake-up satellite $395 \quad(295.5 \mathrm{eV}$ BE, 5.8\%). The C-O-C contribution in the $C 1 s$ XPS line as well as the oxygen content in $O 1 s$ line in the XPS overview spectrum may be explained by the fact that samples have been exposed to the atmospheric air after the graphene growth process when being transferred to the XPS setup. 


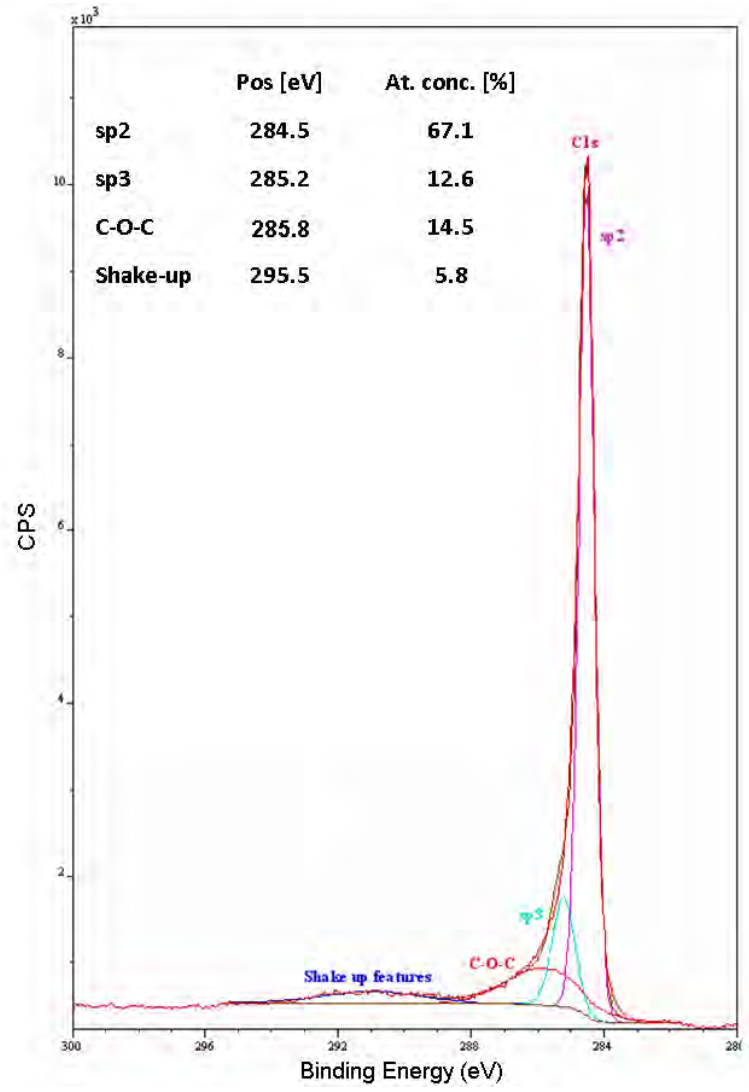

Figure 11: XPS C1s line of the graphene on Ni foil sample 3, and fitting of its different components $\left(s p^{2}, s p^{3}, \mathrm{C}-\mathrm{O}-\mathrm{C}\right.$ and shake up features $)$. Table insert: Measured positions of the XPS lines corresponding to the different components in sample 3, and their relative atomic concentration.

3.2. Catalyst- and field-free growth of vertical graphene sheets on HOPG(0001)

400

We now discuss the rPE-CVD graphene samples 1 and 2 grown onto (0001)oriented HOPG graphite (see Table 1 for deposition parameters).

A clean HOPG(0001) surface layer was generated by Scotch tape cleaving and subsequently loaded into the UHV chamber where it underwent a degassing process at $400^{\circ} \mathrm{C}$ for at least $2 h$. $\mathrm{HOPG}(0001)$ substrates were warmed up to 405 $600^{\circ} \mathrm{C}$ and exposed to $\mathrm{CH}_{4} / \mathrm{H}_{2}$ plasma at $0.2 \mathrm{mbar}$, for 30 and $180 \mathrm{~min}$, with a $\mathrm{RF}$ power of $100 \mathrm{~W}$ ). 
Both samples show a significantly different behaviour in the Raman spectra (see Fig. 12). Sample 2 closely resembles the Raman spectrum of a clean HOPG(0001) substrate (see the Supplementary Content), which gives evidence that there has not been a significant carbon deposition. On the other hand, the 2D peak in sample 1 tends towards the characteristic shape of graphene already observed for the case of the nickel foil substrates: Peak $2 D_{1}$ increasing at the expense of $2 D_{2}$, the $\mathrm{G}$ peak slightly shifted upward $\left(1586 \mathrm{~cm}^{-1}\right)$, together with an extremely large defect peak $\left(\mathrm{I}_{G} / \mathrm{I}_{D}=0.26\right)$ as compared to all previously discussed samples. Due to the large D peak in sample 2, the so-called D' and $\mathrm{D}+\mathrm{D}$ ' peaks also show up, where the D' peak $\left(1620 \mathrm{~cm}^{-1}\right)$ corresponds to a local vibrational mode induced by the high amount of impurities. The latter can interact with the extended phonon modes of graphene, resulting in the splitting of the G peak. [15]

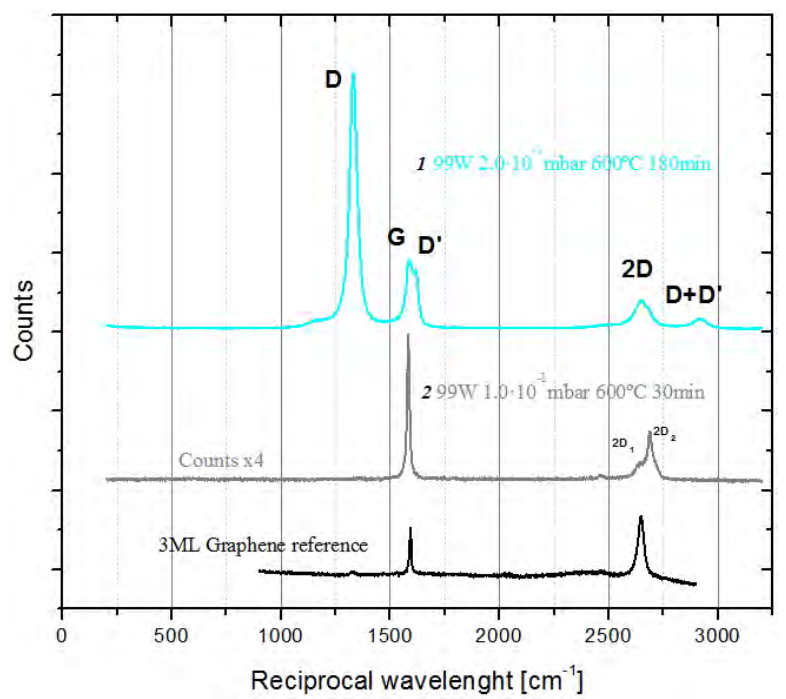

Figure 12: Raman spectra of the collection of rPE-CVD graphene nano-sheet samples 1 and 2 grown on HOPG(0001) (light blue and grey solid line, respectively), together with a 3ML graphene reference sample (black solid line).

In Fig. 13, we show the SEM images of HOPG(0001) samples 1 and 2 as well as the SEM image corresponding to the cleaved pristine HOPG surface. While 


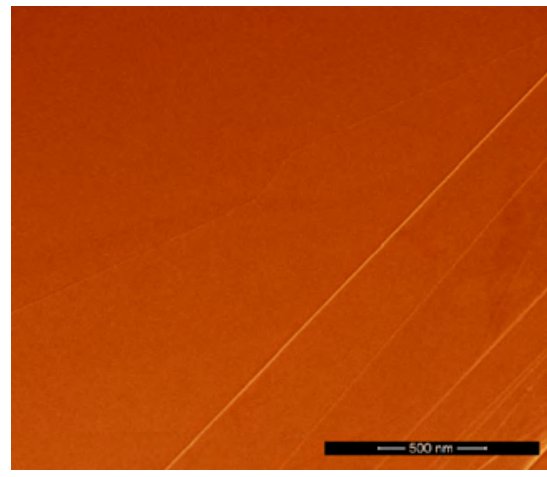

(a) Pristine HOPG(0001)

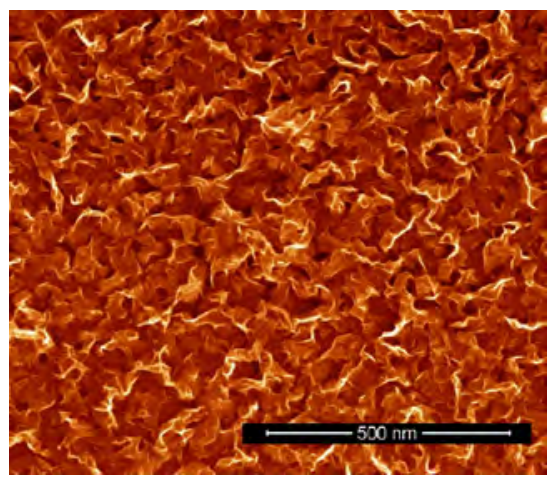

(c) Sample 1

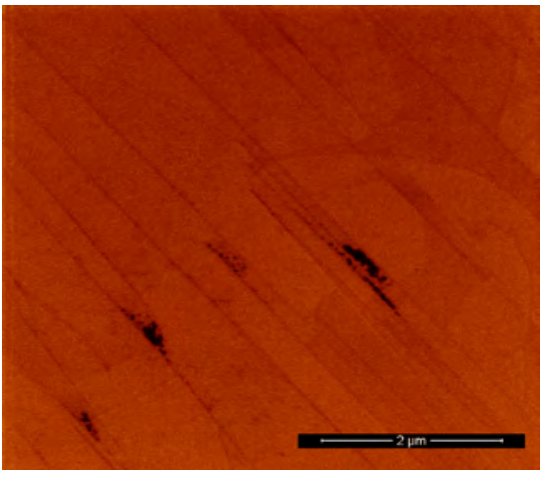

(b) Sample 2

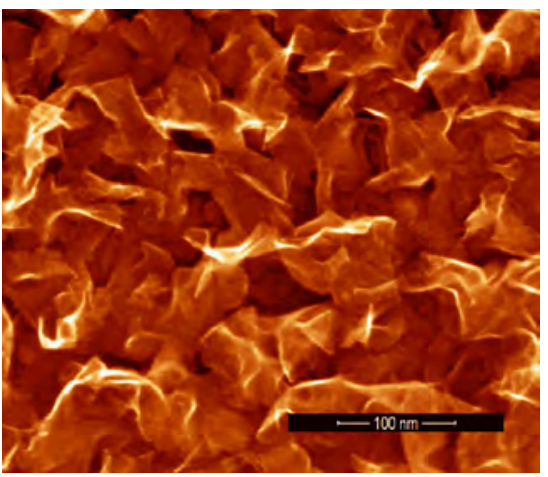

(d) Sample 1 (zoomed in)

Figure 13: SEM images for freshly cleaved HOPG(0001), samples 1 and 2, both grown on HOPG(0001).

the pristine HOPG exhibits the typical remarkably flat, clean, and structureless (exepting a few parallel lines due to step edges) planar appearance, the image of sample $\mathfrak{2}$ gives some additional large features on a several micrometer size scale (see Fig. 13b), which we interpret as large-size two-dimensional graphene layers epitaxially grown onto the pre-existing HOPG(0001) surface. Taking into account the rather long deposition time of 30 min. (e.g., as compared to typical $\mathrm{Ni}$ deposition times) the large size of the observed additional graphene features does not come as a surprise. The SEM image for sample 1 (see Figs. $13 \mathrm{c}$ and ${ }_{430}$ 13d is consistent with the Raman data, as its "vertical nano-sheet" appearance exhibits a considerable amount of sheet edges that largely contribute to the D, 
D', and D+D' peaks. From these SEM images, we estimate the thickness of the graphene nano-sheets to be less than 10 graphene monolayers.

As the growth of graphene on $\mathrm{HOPG}(0001)$ was expected to synthesize planar homo-epitaxial (or at least epitaxial) graphene due to the inherently perfect lattice matching between the $\mathrm{HOPG}(0001)$ and the graphene lattice parameters as can be seen for the case of sample 2 , the above result for sample 1 is surprising. Instead, after a deposition time of $180 \mathrm{~min}$., graphene nano-sheets did grow vertically with respect to the HOPG(0001) surface with random in-plane orientation (see Fig. 13d sample 1), indicating a switch-over of the graphene growth mode between these two samples from in-plane to vertical (see below).

Sample 1 shows a large amount of graphene nano-sheets (Figure 13c) as well as a high defect concentration in peak D of the Raman spectrum (Figure 12. Meanwhile, in the lower growth limit (30 min deposition time), the ${ }_{445}$ Raman spectrum of sample 2 (see Figure 12 gives evidence for a graphene deposition that is structurally identical to the HOPG(0001) substrate, and thus indistinguishable from the Raman spectrum of pristine HOPG (see Fig. ??).

An in-depth analysis of the $C 1 s$ XPS spectrum of sample 1 (see Figure 14) reveals the relative concentration of carbon species (see table in Figure 14). 450 As in the case of the nickel substrates, graphitic carbon $\left(s p^{2}\right.$ at $284.6 \mathrm{eV} \mathrm{BE}$, $61.8 \%)$ is dominant, plus diamond-like carbon $\left(s p^{3}\right.$ at $\left.285.2 \mathrm{eV} \mathrm{BE}, 16.9 \%\right)$, some contribution from oxidized carbon (C-O-C at $286.1 \mathrm{eV} \mathrm{BE}, 9.7 \%$ ), and the $\pi-\pi^{*}$ shake-up satellite (at $289.4 \mathrm{eV} \mathrm{BE}, 11.6 \%$ ). Taking these numbers into account, the C1s XPS spectrum of graphene nano-sheets is closer to that of the early stages of graphene growth on $\mathrm{Ni}(111)$ as compared to the C1s XPS results of graphene on $\mathrm{Ni}$ foil. This can be expected from the large amount of defects and graphene sheet edges visible in the SEM images of both of the former sample systems as compared to the latter graphene sample 3 on $\mathrm{Ni}$ foil.

Comparing the above results with previous studies on the PE-CVD growth of vertical graphene nano-sheets, we would like to point out that this could be achieved without the use of either a surface catalyst or an electric field (perpendicular to the sample surface) as typically generated by the plasma sheath 


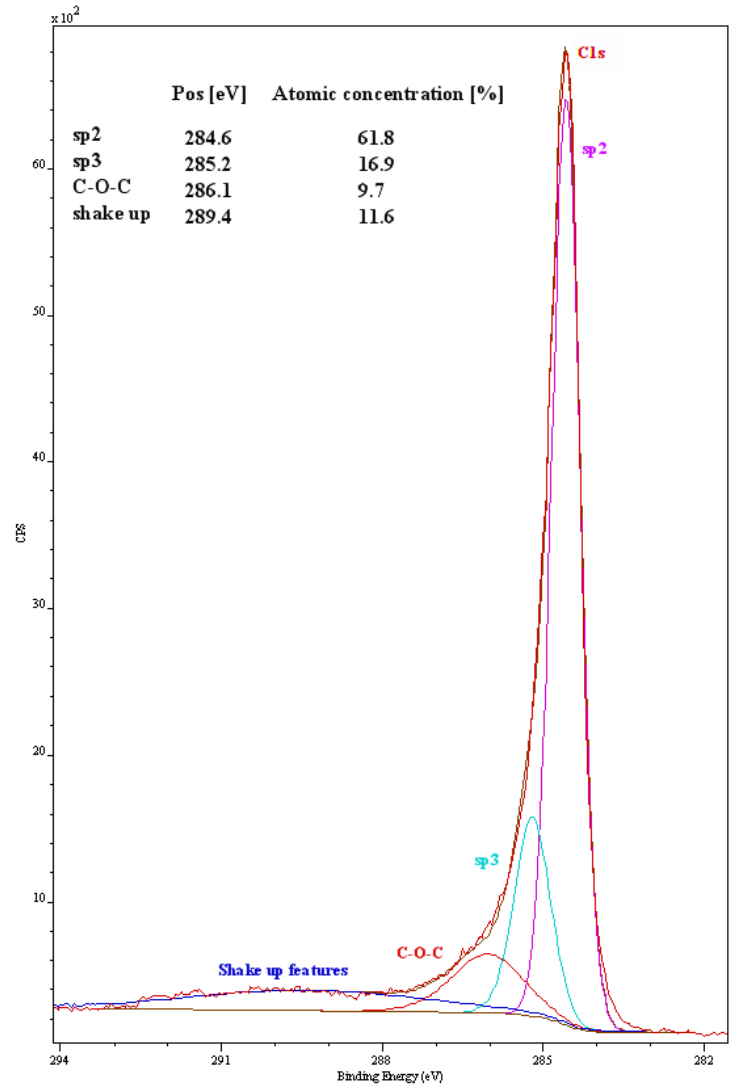

Figure 14: C1s XPS line of the graphene on HOPG(0001) sample 1, and fitting its different components $\left(s p^{2}, s p^{3}, \mathrm{C}-\mathrm{O}-\mathrm{C}\right.$ and shake up features). Insert: XPS overview spectrum of graphene/HOPG(0001) sample 1. Table insert: Measured positions of the peaks corresponding to the different components in sample 1 , and their relative atomic concentration.

and thus inherent to many other commonly used direct plasma PE-CVD setups. While the former catalyst-free nano-sheet growth could already be demonstrated by several groups (see, e.g., [8, 29, 30]), these processes did rely on the inherent occurrence of an electric field as given by the direct sample exposure of the sample within the PE-CVD setup. In our case, the plasma source is remote with respect to the deposition chamber and thus the electromagnetic fields normal to the sample substrate as created by the plasma source - that could possibly induce a preferential growth direction as in the case of a classical CCP or direct 
plasma ICP setup [8] - should be minimized. At this point, the most plausible explanation for this finding is that the upper deposition limit for a $2 \mathrm{D}$ epitaxial deposition was reached for sample 1 due to the extremely long deposition time of 180 minutes, thus leading to a saturation of the $2 \mathrm{D}$ growth parallel to the substrate plane as in HOPG sample 2, thus initiating a vertical growth of graphene nano-sheets. This is corroborated by the experimental findings of Zhu et al. 8, where the authors propose a initial planar graphene growth on $\mathrm{Si}$ and an onset of vertical sheet growth after the deposition of a 1 to $15 \mathrm{~nm}$ flat graphene layer (within $8 \mathrm{~min}$ at $40 \% \mathrm{CH}_{4}$ in $\mathrm{H}_{2}, 100 \mathrm{mTorr}, 680^{\circ} \mathrm{C}$ sample temperature, and $900 \mathrm{~W}$ RF power), when a sufficient level of force develops at the graphene sheet boundaries to curl the leading edge of the top layers upward. Their underlying growth model is based on the production of atomic hydrogen in their ICP plasma, plus the above vertical electric field in conjunction with the polarizability of the graphene sheets. The plasma feedstock gas mixture and substrate temperature used in our experiments do bear some similarity in view of the plasma chemistry, whereas the deposition pressure and RF power are at variance by about one order of magnitude - which may account for the large variation in deposition time (i.e., 8 min versus $180 \mathrm{~min}$ ). Nevertheless, the major difference is given by the occurrence (or non-occurrence) of a significant perpendicular electric field, thus suggesting a different mechanism underlying the initiation of the vertical growth mode as the one proposed by Zhu et al. [8].

\section{Conclusions}

We have successfully implemented and used a newly developed remote inductively coupled low-pressure RF plasma source for the synthesis of multilayer micro-crystalline graphene on polycrystalline nickel foil and $\mathrm{Ni}(111)$ by means of remote plasma-enhanced chemical vapor deposition (rPE-CVD). Also, the catalyst- and field-free growth of vertical graphene nano-sheets has been achieved on HOPG(0001).

For the graphene/Ni foil samples, Raman spectroscopy revealed the pre- 
dominance of an ordered $s p^{2}$ growth process with an extremely low amount of defects over a wide surface area, as evidenced by sharp G and D peaks as well as STM analysis. Raman spectra also did yield a first qualitative evidence of the multilayer nature of our samples, as witnessed by the low $2 \mathrm{D} / \mathrm{G}$ ratio. The graphene thickness was quantitatively established to be close to six graphene monolayers, using the ratio between Ni2p and C1s peaks in the XPS spectra. On $\mathrm{Ni}$ foils, the addition of a small amount $(\sim 20 \%)$ of $\mathrm{H}_{2}$ into the plasma feedstock gas mixture turns out to be crucial to reduce the growth of a disordered $s p^{3}$ or amorphous $s p^{3} / s p^{2}$ carbon phases. So far, substrate temperatures slightly above $700^{\circ} \mathrm{C}$ and $\mathrm{RF}$ powers of $\sim 200 \mathrm{~W}$ have proven to be optimum, in contrast to lower values that lead to an insufficient growth rate for the specific case of a Ni foil substrate. Moreover, a deposition time of 3 to $5 \mathrm{~min}$ for 6 graphene monolayers at gas pressures of 0.1 mbar did yield our best results in view of the growth of defect-free large area graphene layers. Lower deposition times are inefficient, whereas lower pressures do enhance the deposition rate, thereby resulting in an excessive growth of thick graphene films together with an enhanced density of defects.

Polycrystalline $\mathrm{Ni}$ foils have so far been the most promising substrates to perform plasma-assisted depositions of graphene, as they yield a growth of 2dimensional multilayers. Even though we have so far not tested a fine control over the number of deposited layers, this could be easily achieved in the near future by means of performing dedicated deposition runs following the already established procedures. The rPE-CVD graphene samples synthesized on the Ni foils have proven to be good candidates for STM analysis, yielding a fairly complete picture of the produced layers via the imaging of the carbon atoms contained in the graphene lattice. In more detail, large defect-free graphene areas could be demonstrated together with smooth transitions between substrate defects as well as areas with different graphene stacking modes.

A catalyst- and field-free homogeneous coverage of vertical graphene nanosheets was successfully achieved on $\operatorname{HOPG}(0001)$. To our knowledge, this could be achieved under catalyst- and field-free conditions for the 
first time. SEM images did show a clear perpendicular growth due to a combination of deposition rate and extended deposition time, resulting into the aforementioned vertical graphene nano-sheets. Based on a comparison with similar results from the PE-CVD literature, we would like to point out the existence of a transition from an in-plane to a vertical growth mode together with the use of atomic hydrogen within the plasma. However, we see no direct evidence for the need of significant electric fields perpendicular to the sample surface or of surface catalysts to enable the growth of vertical graphene nano-sheets. Working on the hypothesis of long deposition times leading to a vertical growth, further $\mathrm{rPE}-\mathrm{CVD}$ studies are needed in order to elucidate the detailed dynamics regarding the transition between the in-plane and the vertical growth modes of graphene nano-sheets on HOPG(0001).

Summarizing the above results, the GV10x rPE-CVD downstream (or afterglow) plasma source appears to be a promising tool for the deposition of graphene micro- and nanostructures as well as for the more complex in situ UHV preparation of, e.g., multi-layered hybrid graphene sample systems.

\section{Acknowledgments}

We acknowledge support by expert staff of ibss Group Inc. We also acknowledge the scientific advice by S. Ferrer (CELLS) and the contribution by the CELLS technical staff, especially L. Ginés, as well as the friendly collaboration of M.Panighel, N.Domingo, and A.Verdaguer (ICN2) during the sample characterization activities. A. R. G. thanks the Spanish Ministry of Economy and Competitiveness (MINECO) for its support through Grant No. CSD2010-00044

(Consolider NANOTHERM). The work at ICMAB (Raman measurements) was carried out under the auspices of the Spanish Severo Ochoa Centre of Excellence program (grant SEV-2015-0496). SEM, STM, and XPS measurements were performed at ICN2 that acknowledges support from the Severo Ochoa Program (MINECO, Grant SEV-2013-0295). PEEM and LEEM measurements 
with the assistance of CELLS-ALBA staff. E. Pach is enrolled in the PhD program in Materials Sciences at the UAB.

\section{References}

[1] Y. B. Zhang, Y. W. Tan, H. L. Stormer, P. Kim, Experimental observation of the quantum Hall effect and Berry's phase in graphene, Nature 438 (7065) (2005) 201-204. doi:10.1038/nature04235.

[2] T. Ohta, Controlling the Electronic Structure of Bilayer Graphene, Science 313 (5789) (2006) 951-954. doi:10.1126/science.1130681.

[3] D. Boyd, W.-H. Lin, C.-C. Hsu, M. Teague, C.-C. Chen, Y.-Y. Lo, W.-Y. Chan, W.-B. Su, T.-C. Cheng, C.-S. Chang, C.-I. Wu, N.-C. Yeh, Singlestep deposition of high-mobility graphene at reduced temperatures, Nature Communications 6 (2015) 6620. doi:10.1038/ncomms7620

URL http: //www . nature.com/doifinder/10.1038/ncomms7620

[4] G. Nandamuri, S. Roumimov, R. Solanki, Remote plasma assisted growth of graphene films, Appl. Phys. Lett. 96 (15) (2010) 154101. doi:10.1063/ 1.3387812 .

[5] J. Kim, M. Ishihara, Y. Koga, K. Tsugawa, M. Hasegawa, S. Iijima, Lowtemperature synthesis of large-area graphene-based transparent conductive films using surface wave plasma chemical vapor deposition, Appl. Phys. Lett. 98 (9) (2011) 091502. doi:10.1063/1.3561747.

[6] L. Zhang, Z. Shi, Y. Wang, R. Yang, D. Shi, G. Zhang, Catalyst-free growth of nanographene films on various substrates, Nano Res. 4 (3) (2011) 315321. doi:10.1007/s12274-010-0086-5.

[7] L. Zhang, Z. Shi, D. Liu, R. Yang, D. Shi, G. Zhang, Vapour-phase 585 graphene epitaxy at low temperatures, Nano Res. 5 (4) (2012) 258-264. doi:10.1007/s12274-012-0205-6. 
[8] M. Zhu, J. Wang, B. C. Holloway, R. Outlaw, X. Zhao, K. Hou, V. Shutthanandan, D. M. Manos, A mechanism for carbon nanosheet formation, Carbon N. Y. 45 (11) (2007) 2229-2234. doi:10.1016/j.carbon.2007. 06.017

[9] CELLS - ALBA Synchrotron Light Source,

URL http://www.cells.es

[10] ibss Group Inc., URL http;//www.ibssgroup.com.

[11] E. Pellegrin, I. Šics, J. Reyes-Herrera, C. Perez Sempere, J. J. Lopez Alcolea, M. Langlois, J. Fernandez Rodriguez, V. Carlino, Characterization, optimization and surface physics aspects of in situ plasma mirror cleaning, J. Synchrotron Radiat. 21 (2) (2014) 300-314. doi:10.1107/ S1600577513032402.

[12] M. G. Cuxart, J. Reyes-Herrera, I. Šics, A. R. Goñi, H. M. Fernandez, V. Carlino, E. Pellegrin, Remote plasma cleaning of optical surfaces: Cleaning rates of different carbon allotropes as a function of RF powers and

q distances, Appl. Surf. Sci. 362 (2016) 448-458. doi:10.1016/j.apsusc. 2015.11.117.

[13] A. Fridman, L. A. Kennedy, Plasma Physics and Engineering, 2011.

[14] Alexander Fridman, Plasma Chemistry, 2012.

[15] A. C. Ferrari, J. C. Meyer, V. Scardaci, C. Casiraghi, M. Lazzeri, F. Mauri, S. Piscanec, D. Jiang, K. S. Novoselov, S. Roth, A. K. Geim, Raman Spectrum of Graphene and Graphene Layers, Phys. Rev. Lett. 97 (18) (2006) 187401. doi:10.1103/PhysRevLett.97.187401.

[16] PLASUS Specline, PLASUS GmbH, Mering, Germany.

[17] W. S. W. S. M. Werner, C. J. Powel, NIST Database for the Simulation of Electron Spectra for Surface Analysis (SESSA), National Institute of Standards and Technology, Gaithersburg, Maryland. 
[18] A. C. Ferrari, D. M. Basko, Raman spectroscopy as a versatile tool for studying the properties of graphene, Nat. Nanotechnol. 8 (4) (2013) 235246. doi:10.1038/nnano.2013.46.

[19] C. Thomsen, S. Reich, Double Resonant Raman Scattering in Graphite, Phys. Rev. Lett. 85 (24) (2000) 5214-5217. doi:10.1103/PhysRevLett. 85.5214

[20] A. C. Ferrari, Raman spectroscopy of graphene and graphite: Disorder, electron-phonon coupling, doping and nonadiabatic effects, Solid State Commun. 143 (1-2) (2007) 47-57. doi:10.1016/j.ssc.2007.03.052.

[21] A. Reina, X. Jia, J. Ho, D. Nezich, H. Son, V. Bulovic, M. S. Dresselhaus, J. Kong, Large Area, Few-Layer Graphene Films on Arbitrary Substrates 625 \ by Chemical Vapor Deposition 2009, Nano Lett. 9 (2009) 30-35. doi: $10.1021 / \mathrm{n} 1801827 \mathrm{v}$.

[22] Z. R. Robinson, P. Tyagi, T. M. Murray, C. A. Ventrice, S. Chen, A. Mun-

a son, C. W. Magnuson, R. S. Ruoff, Substrate grain size and orientation of cu and cu-ni foils used for the growth of graphene films, Journal of

630 Vacuum Science \& Technology A 30 (1) (2012) 23919-23927. doi:http: $/ / \mathrm{dx} . \mathrm{doi}$. org/10.1116/1.3663877

a URL http://scitation.aip.org/content/avs/journal/jvsta/30/1/ $10.1116 / 1.3663877$

[23] A. C. Ferrari, J. Robertson, Raman spectroscopy of amorphous, nanostructured, diamond-like carbon, and nanodiamond, Philos. Trans. R. Soc.

口 A Math. Phys. Eng. Sci. 362 (1824) (2004) 2477-2512. doi:10.1098/rsta. 2004.1452 .

[24] NIST Atomic Spectra Database Version 2.0., Gaithersburg, Maryland, National Institute of Standards and Technology.

${ }_{640}[25]$ T. L. Burnett, R. Yakimova, O. Kazakova, Identification of epitaxial graphene domains and adsorbed species in ambient conditions using quan- 
tified topography measurements, J. Appl. Phys. 112 (5) (2012) 054308. doi:10.1063/1.4748957.

[26] F. Bianchini, L. L. Patera, M. Peressi, C. Africh, G. Comelli, Atomic Scale Identi fi cation of Coexisting Graphene Structures on Ni(111), J. Phys. Chem. Lett. (5) (2014) 467-473.

[27] W. Yan, L. Meng, M. Liu, J.-B. Qiao, Z.-D. Chu, R.-F. Dou, Z. Liu, J.-C. Nie, D. G. Naugle, L. He, Angle-dependent van hove singularities and their breakdown in twisted graphene bilayers, Phys. Rev. B 90 (2014) 115402. doi:10.1103/PhysRevB.90.115402

URL http://link.aps .org/doi/10.1103/PhysRevB.90.115402

[28] I. Horcas, R. Fernandez, J. M. Gomez-Rodriguez, J. Colchero, J. GomezHerrero, A. M. Baro, Wsxm: A software for scanning probe microscopy and a tool for nanotechnology, Review of Scientific Instruments 78 (1). doi:http://dx.doi.org/10.1063/1.2432410.

a URL http://scitation.aip.org/content/aip/journal/rsi/78/1/10. $1063 / 1.2432410$

[29] J. Wang, M. Zhu, R. Outlaw, X. Zhao, D. Manos, B. Holloway, Synthesis of carbon nanosheets by inductively coupled radio-frequency plasma enhanced chemical vapor deposition, Carbon N. Y. 42 (14) (2004) 2867-2872. doi: $10.1016 / \mathrm{j}$.carbon.2004.06.035.

[30] J. J. Wang, M. Y. Zhu, R. A. Outlaw, X. Zhao, D. M. Manos, B. C. Holloway, V. P. Mammana, Free-standing subnanometer graphite sheets, Applied Physics Letters 85 (7) (2004) 1265-1267. doi:http://dx.doi. org/10.1063/1.1782253.

口 URL http://scitation.aip.org/content/aip/journal/apl/85/7/10. $1063 / 1.1782253$ 
Fig. 1

Click here to download high resolution image
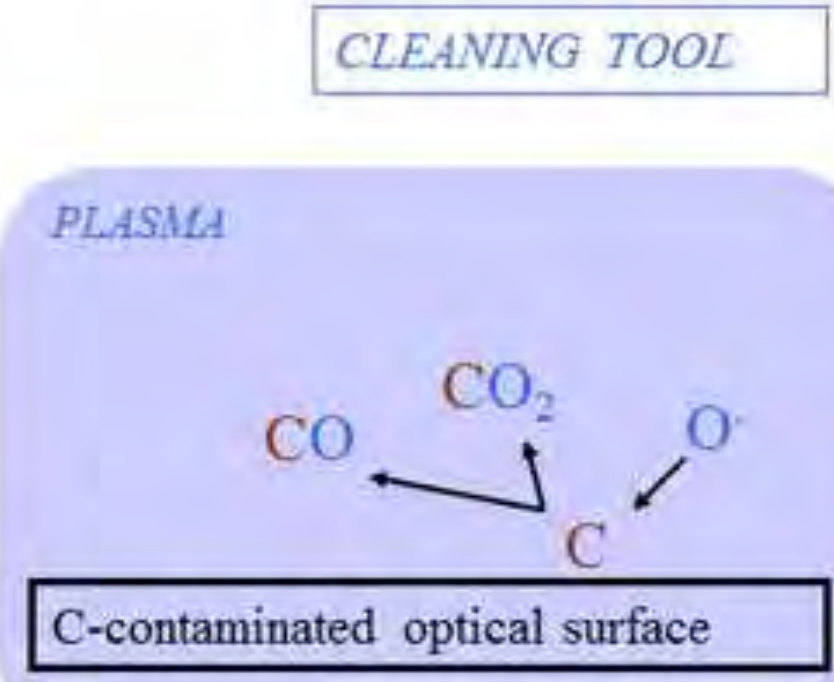

DEPOSITION TOOL

$\longrightarrow$ rPECVD
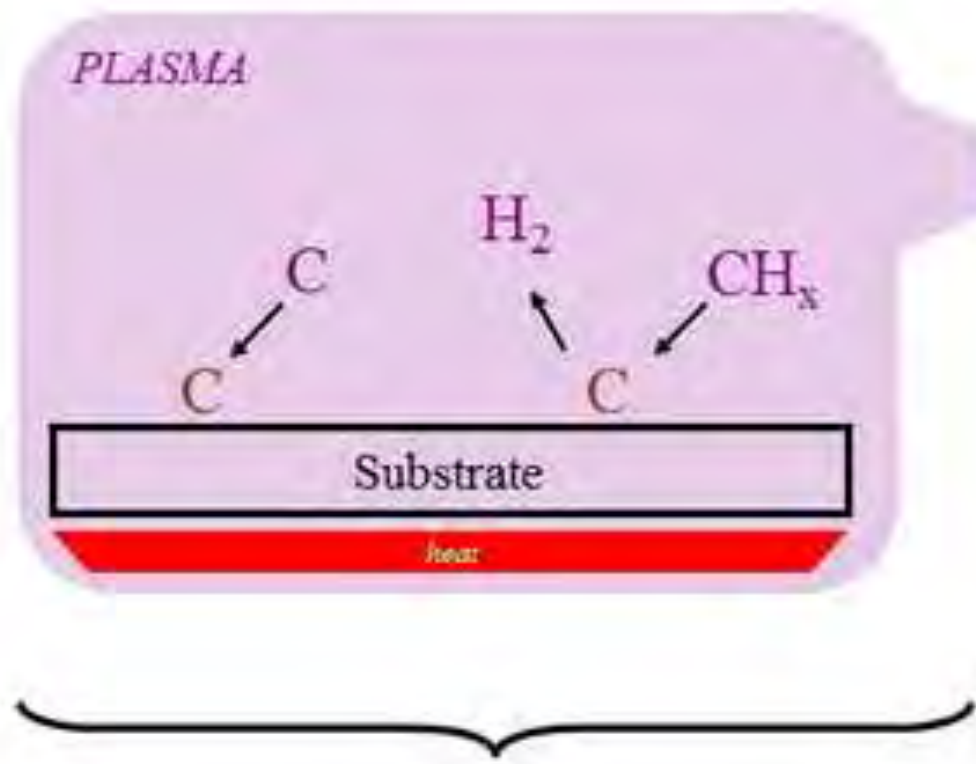

Chemical activity
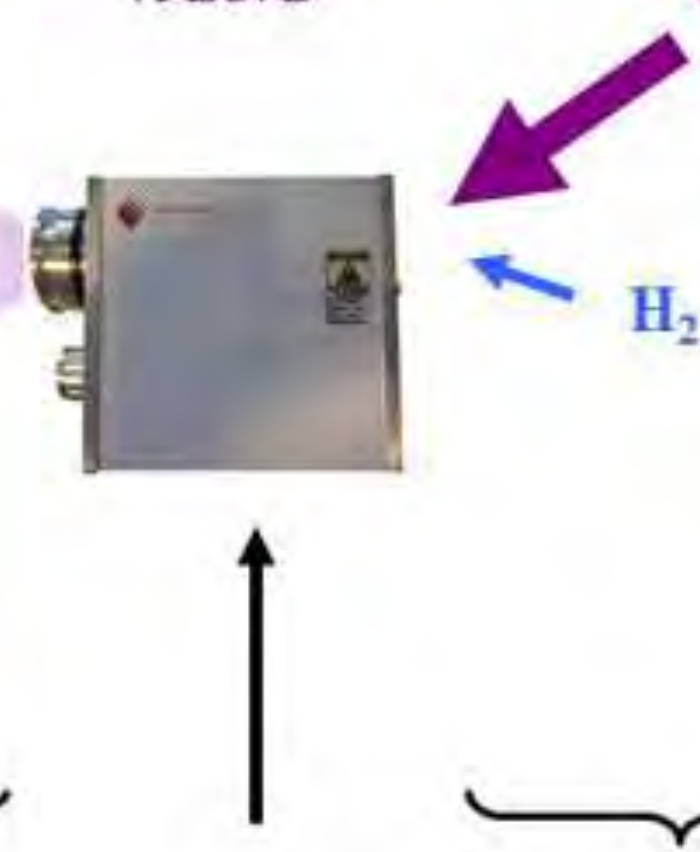

GV10x

Feedstock 
Fig. 2

Click here to download high resolution image
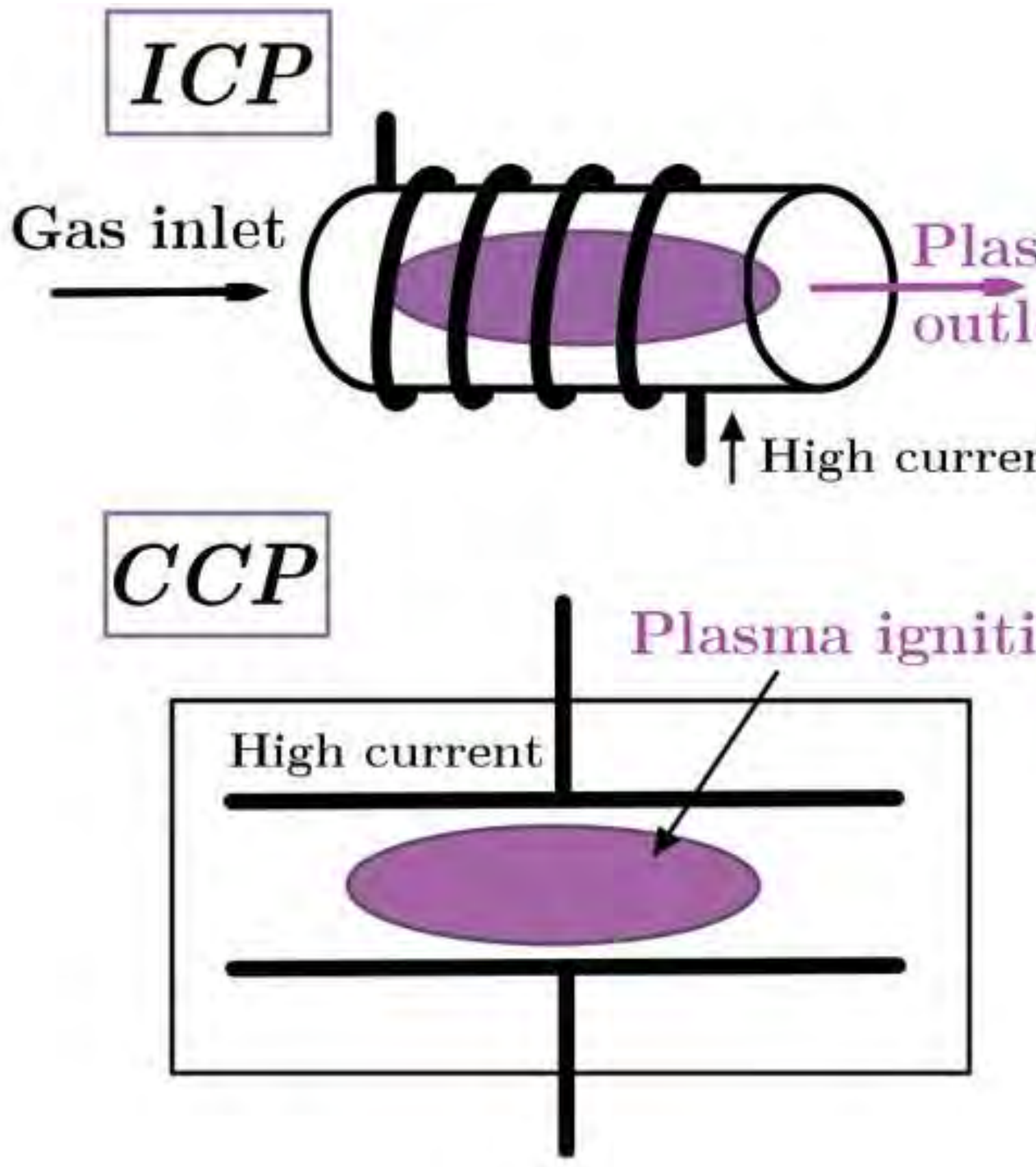


\section{Fig. 3}

Click here to download high resolution image

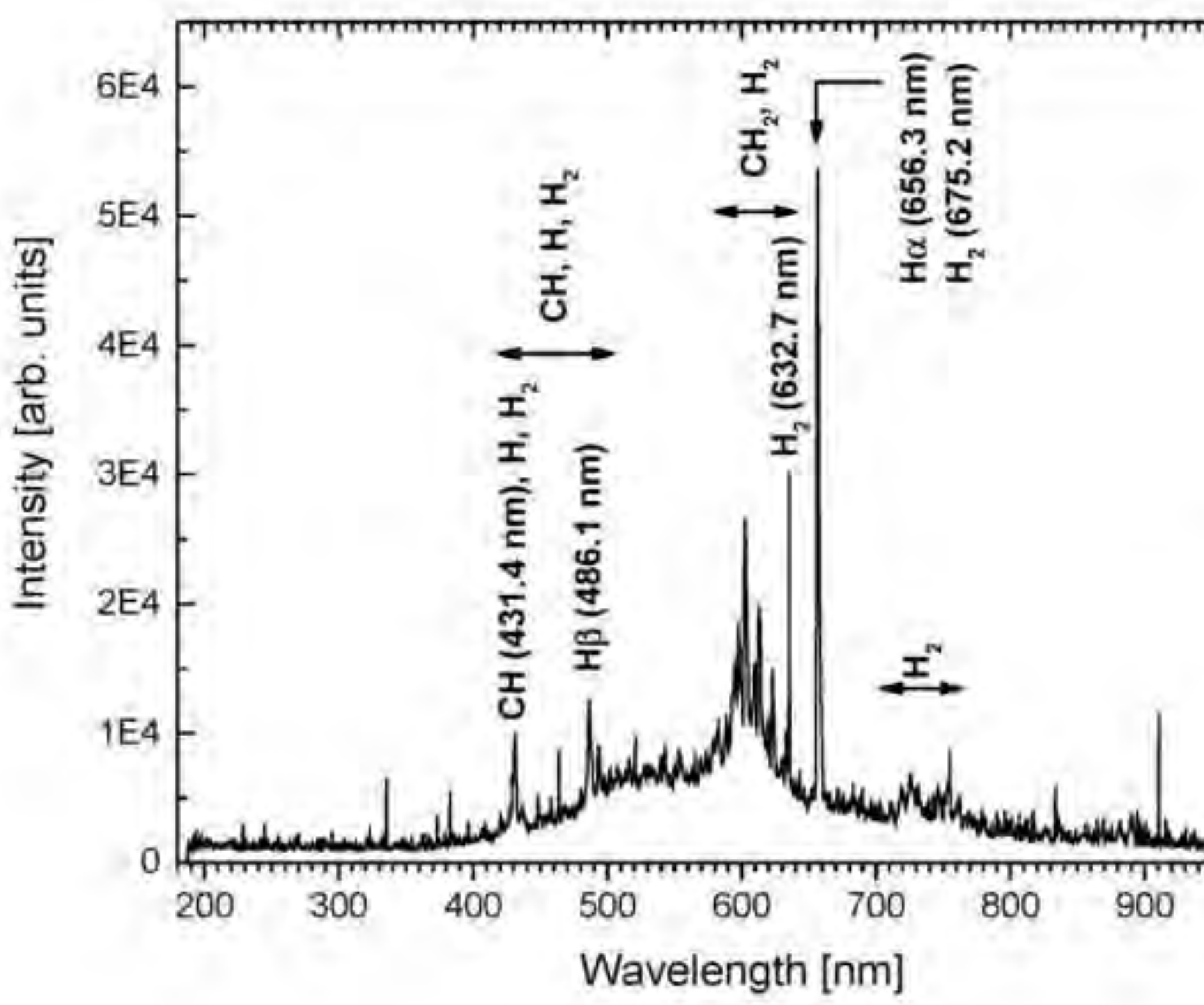


Fig. $4 a$

Click here to download high resolution image

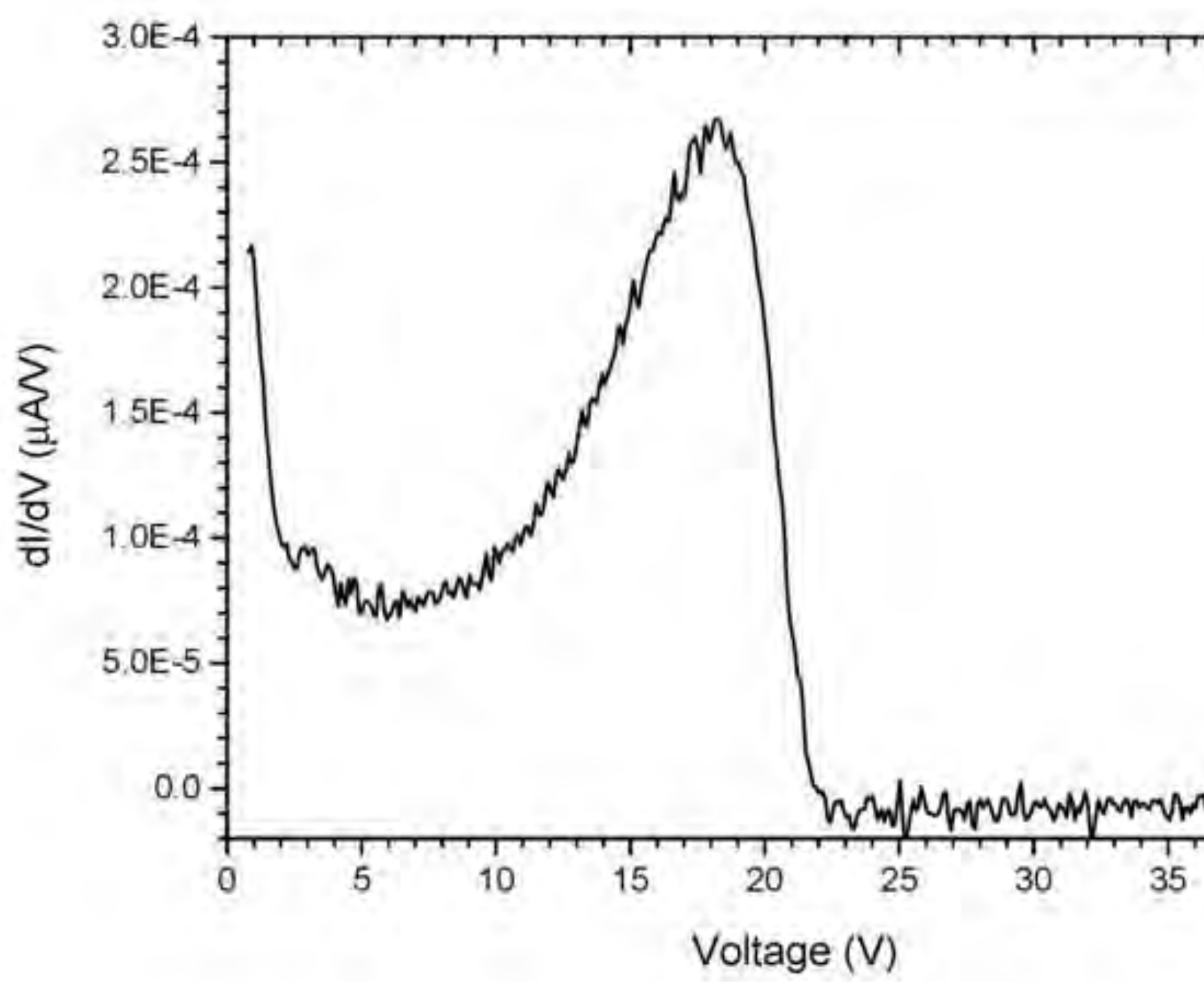




\section{Fig. $4 b$}

Click here to download high resolution image

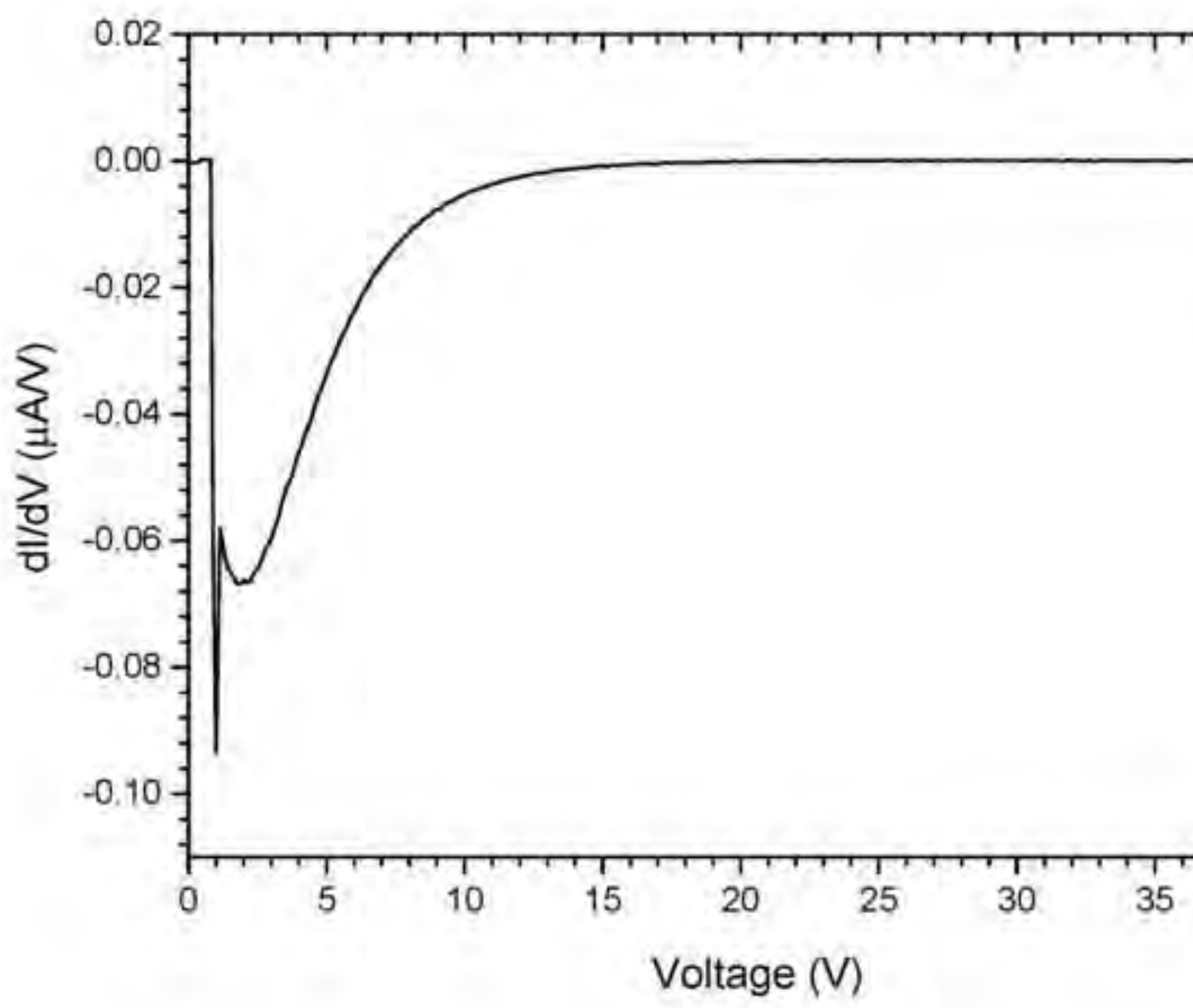




\section{Fig. 5}

Click here to download high resolution image

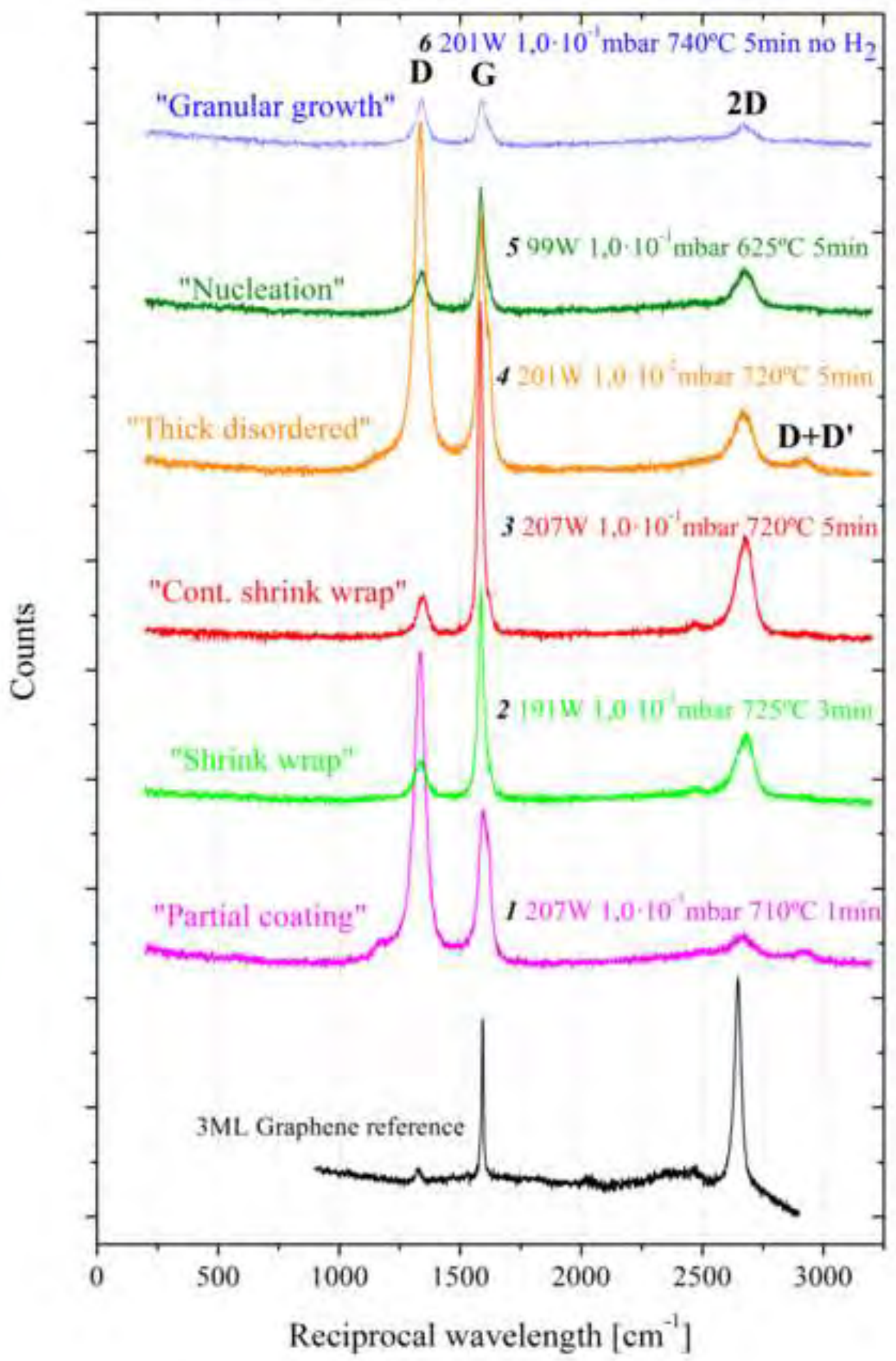


Fig. $6 a$
Click here to download high resolution image

Fig. $6 a$
Click here to download high resolution image

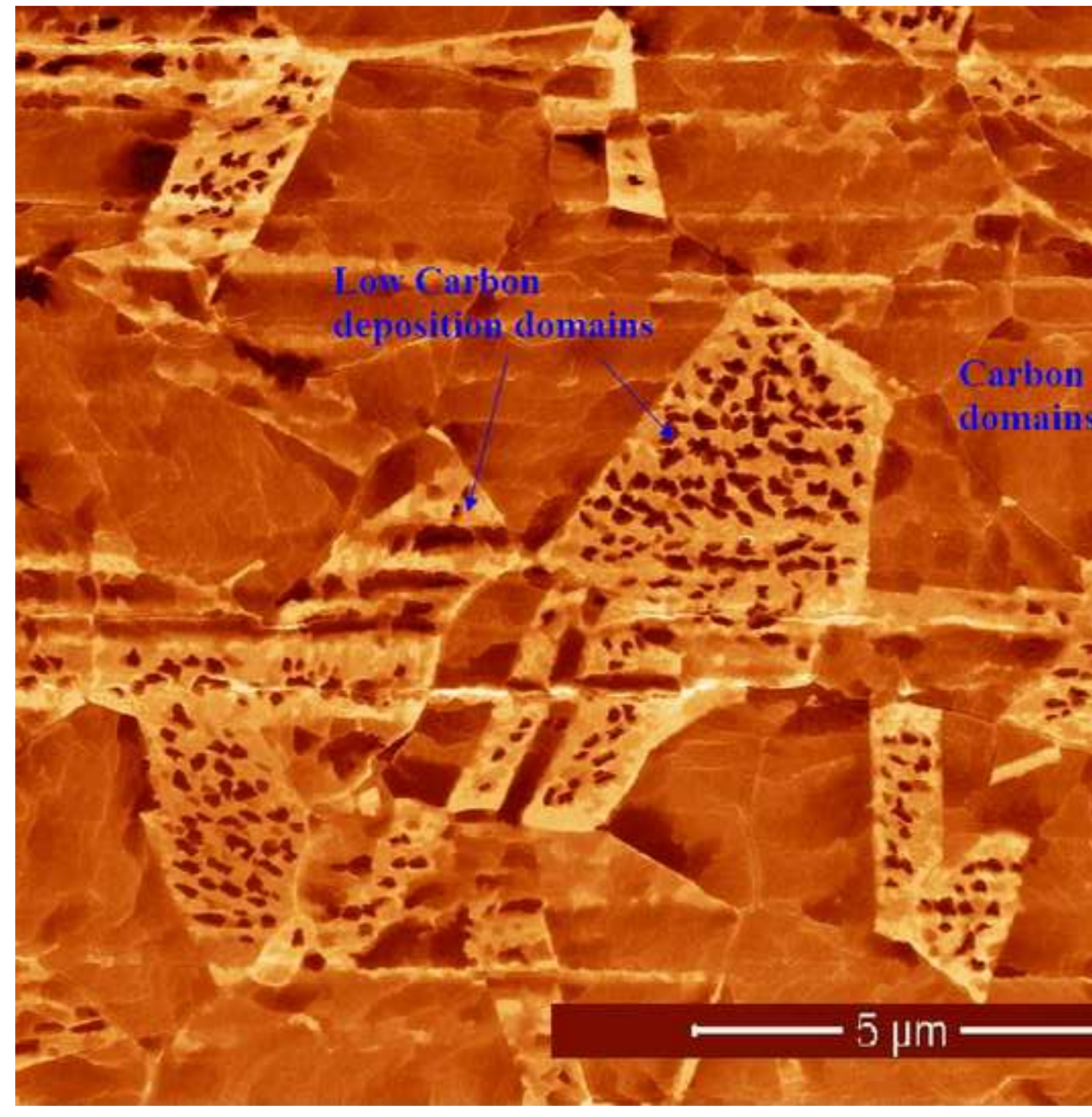


Click here to download high resolution image

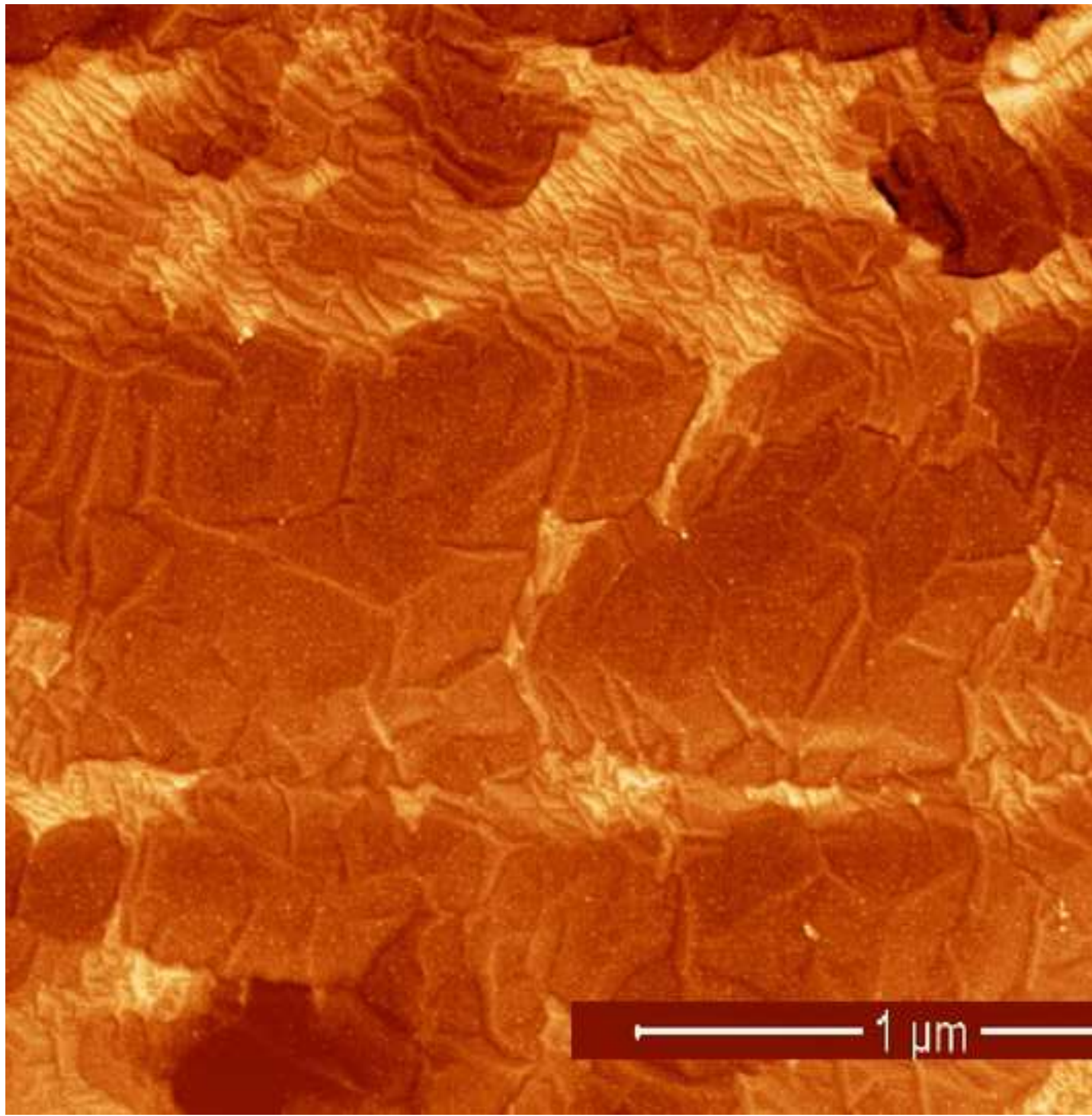


Fig. 6c

Click here to download high resolution image

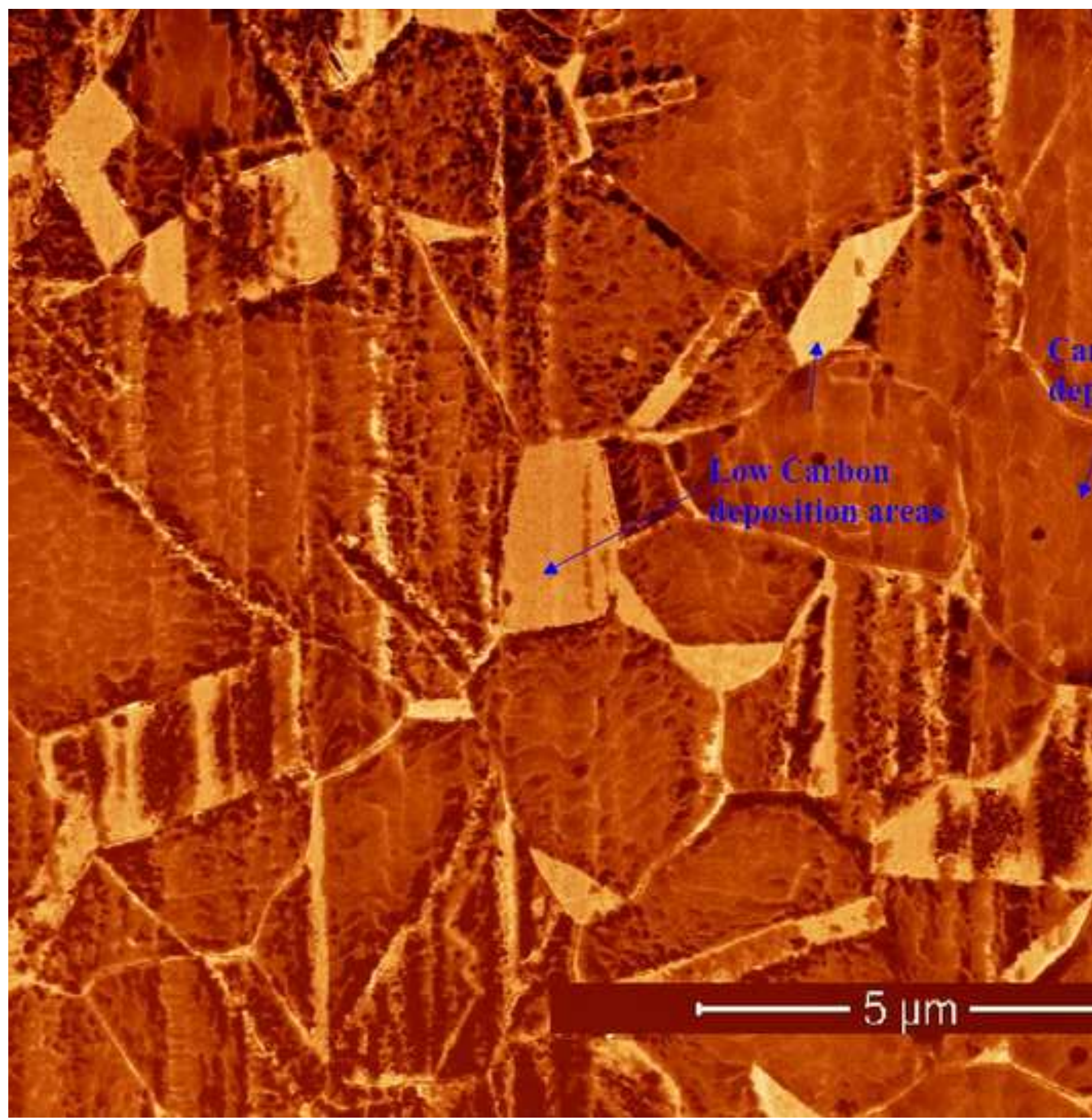


Click here to download high resolution image
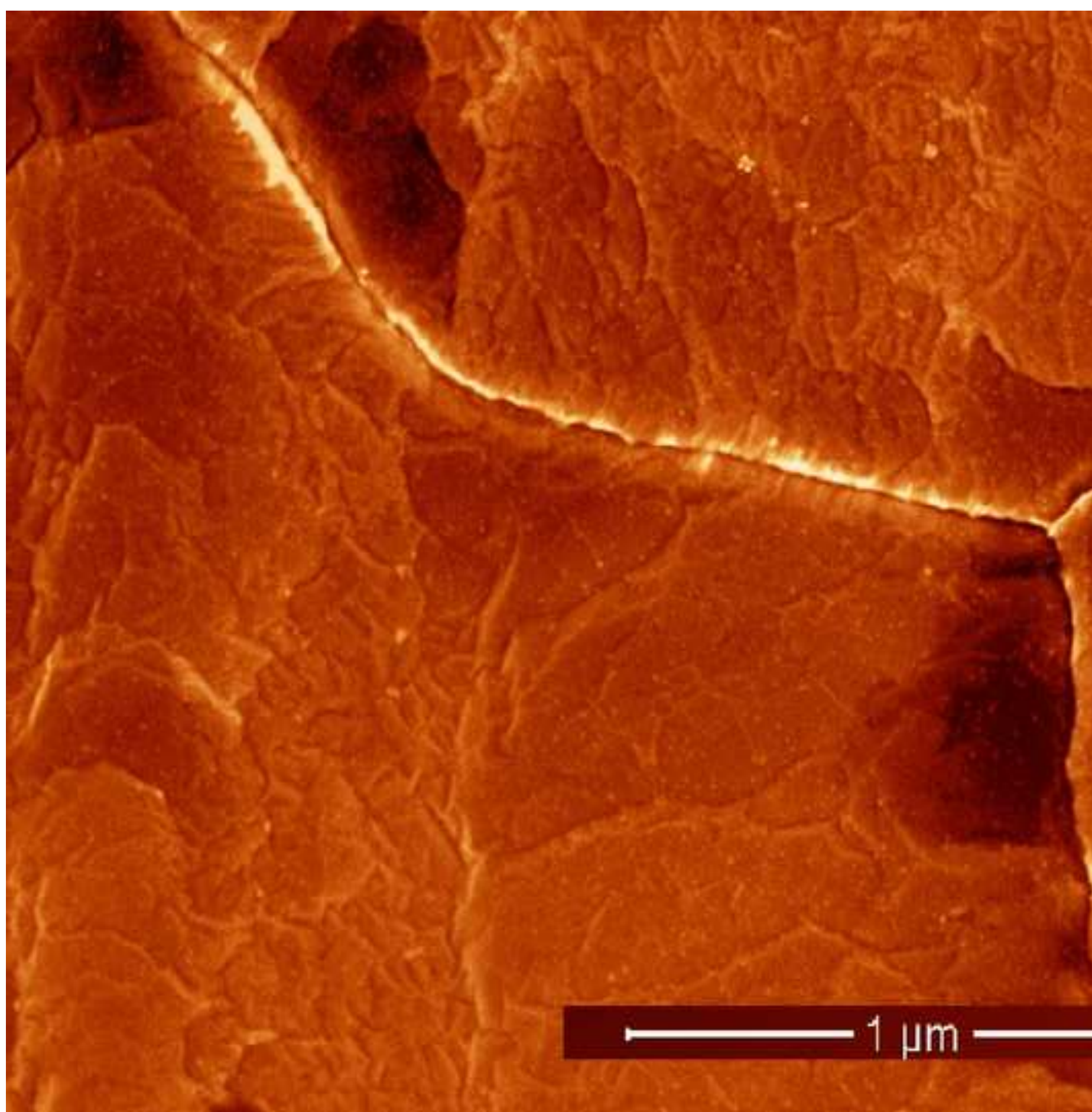
Fig. $6 \mathrm{e}$
Click here to download high resolution image

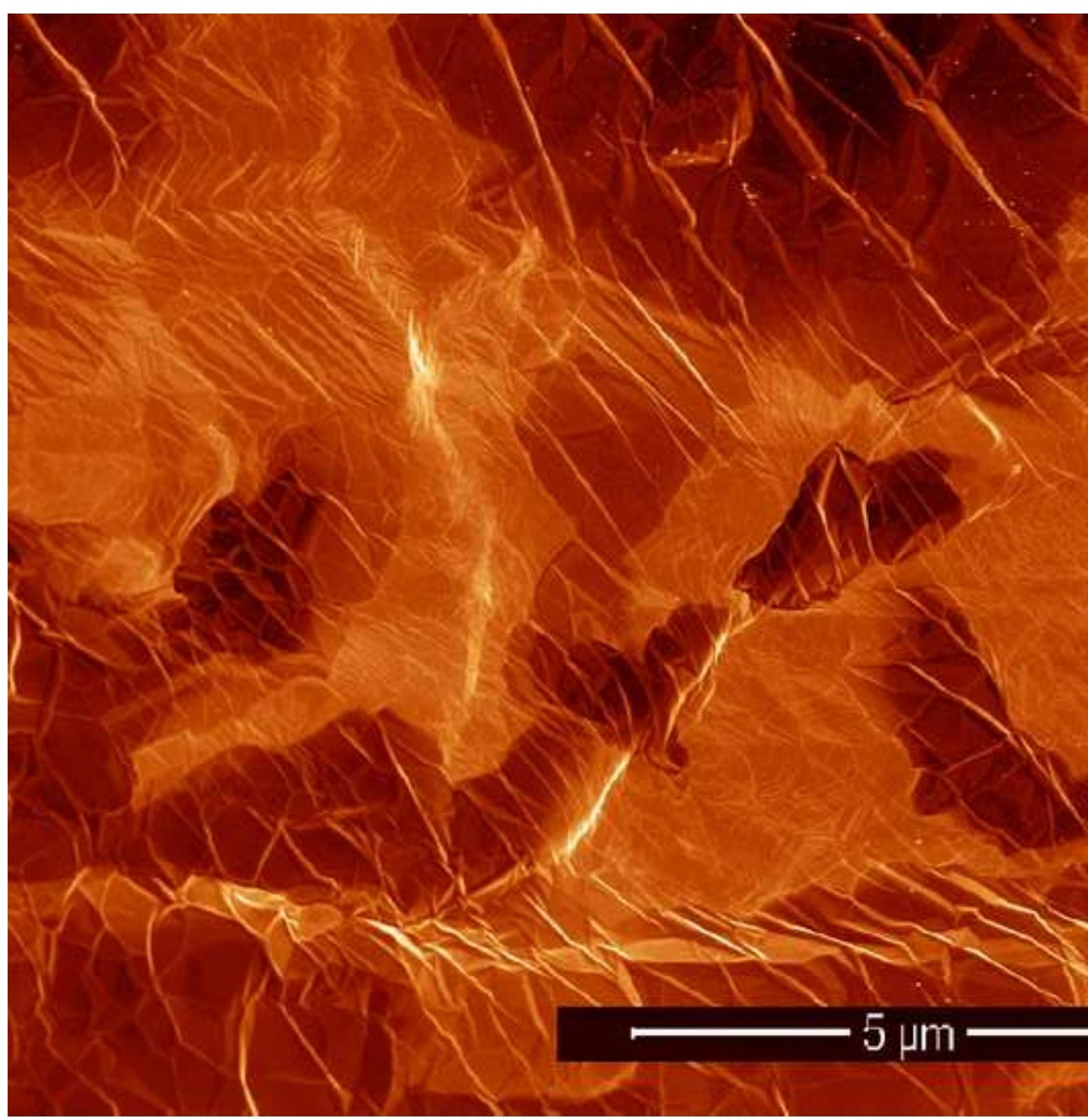


Click here to download high resolution image

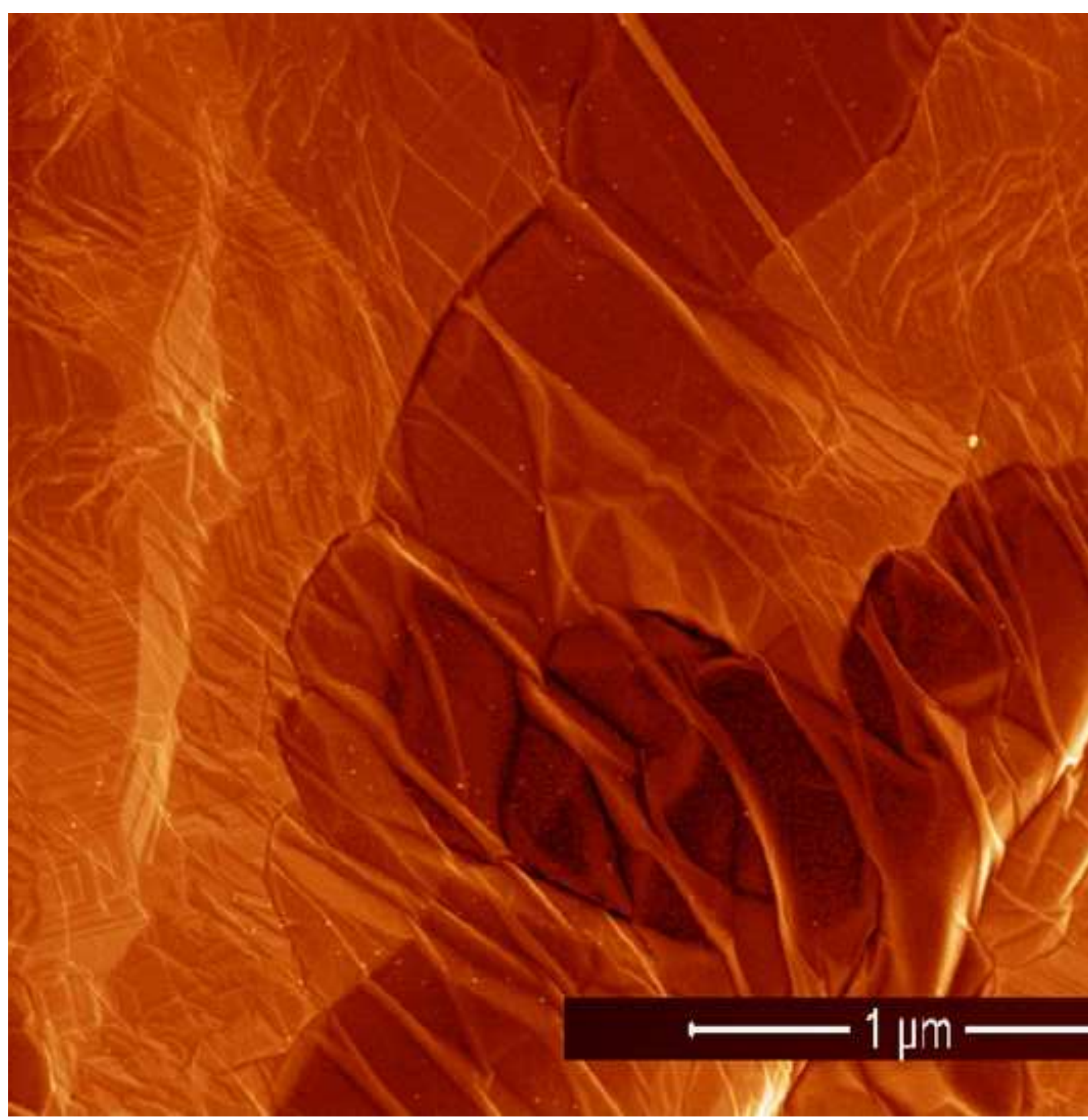


Click here to download high resolution image

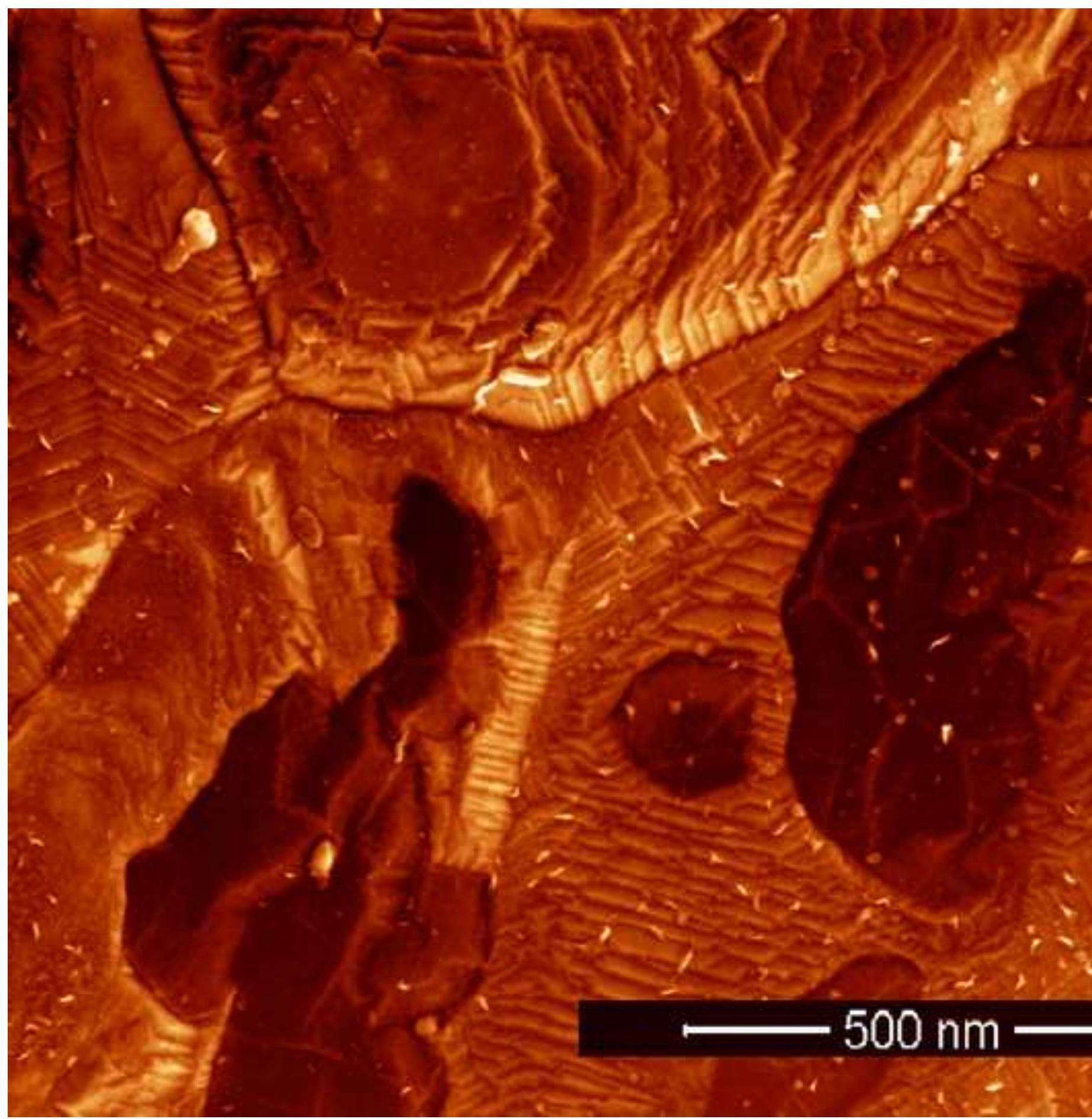


Click here to download high resolution image

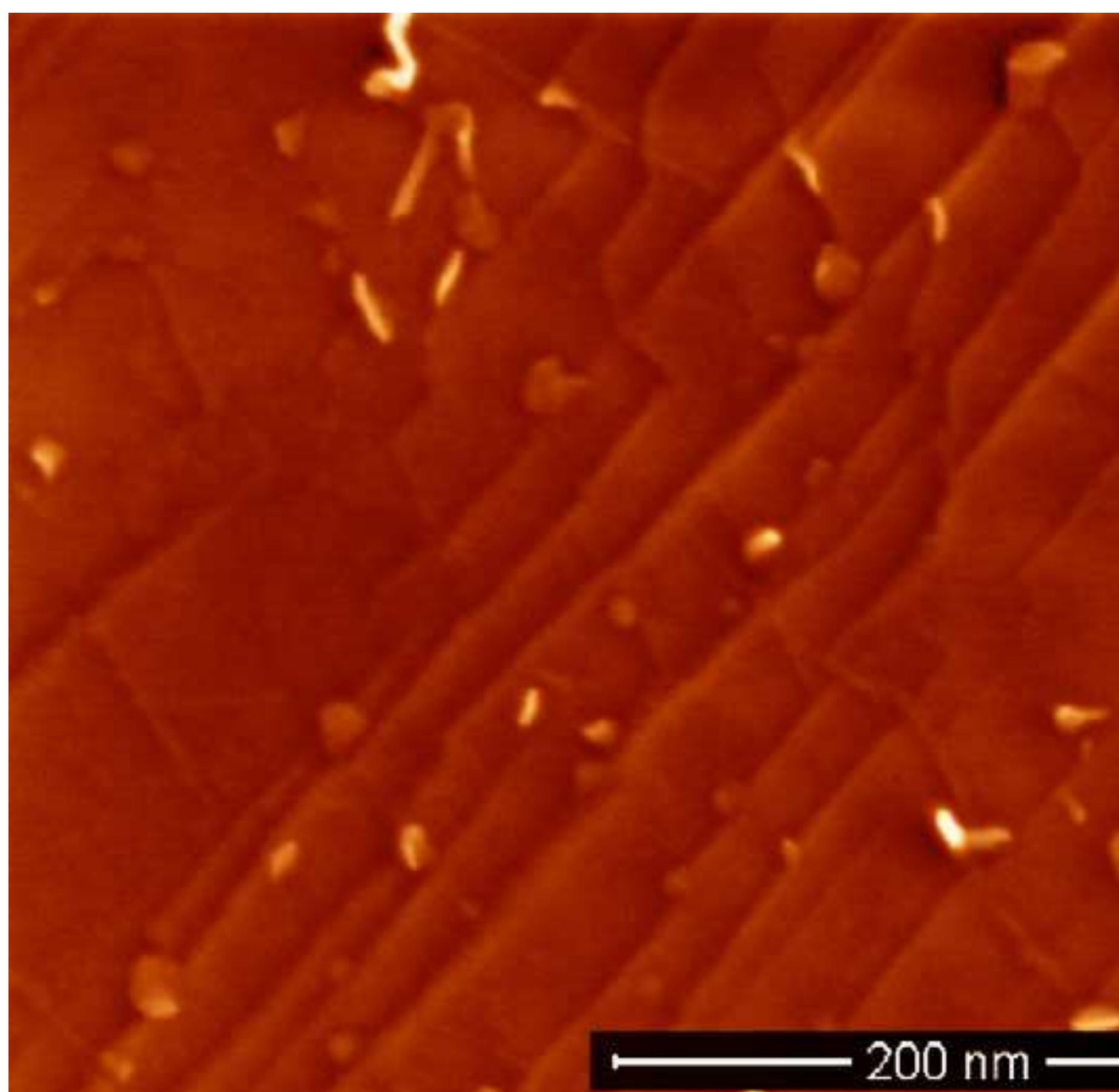


Click here to download high resolution image

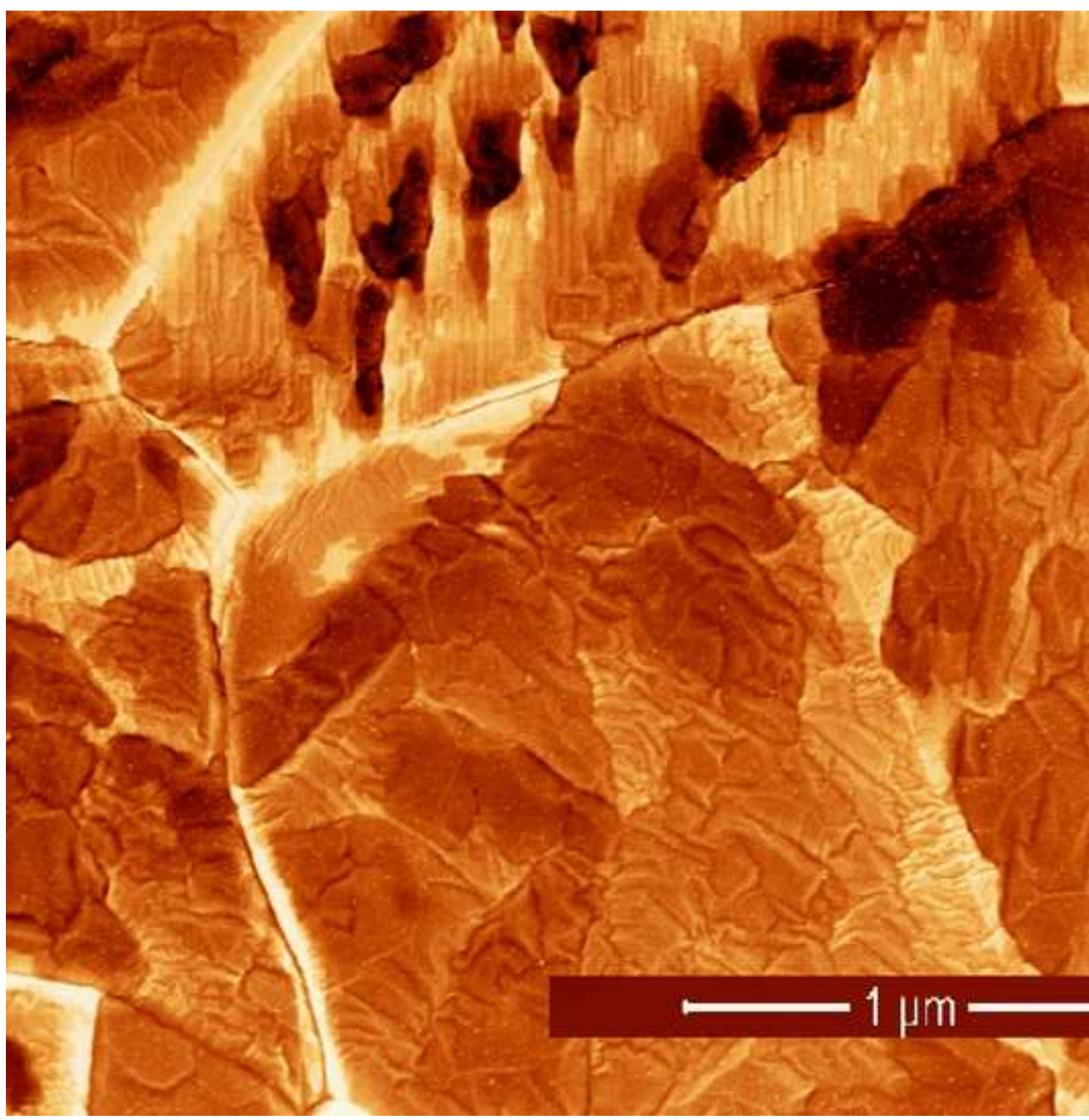


Fig. $7 d$
Click here to download high resolution image

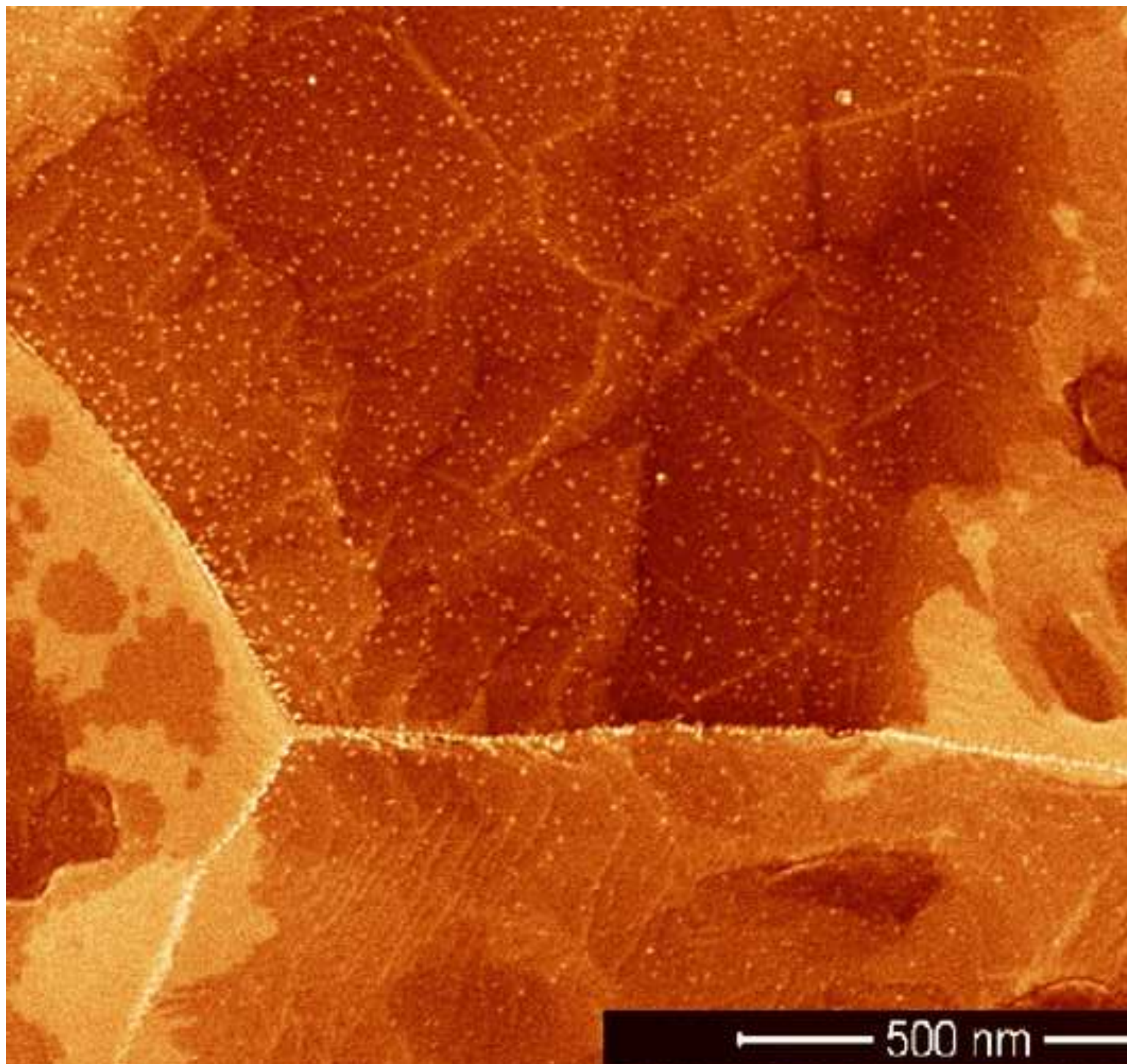


Fig. 7e

Click here to download high resolution image

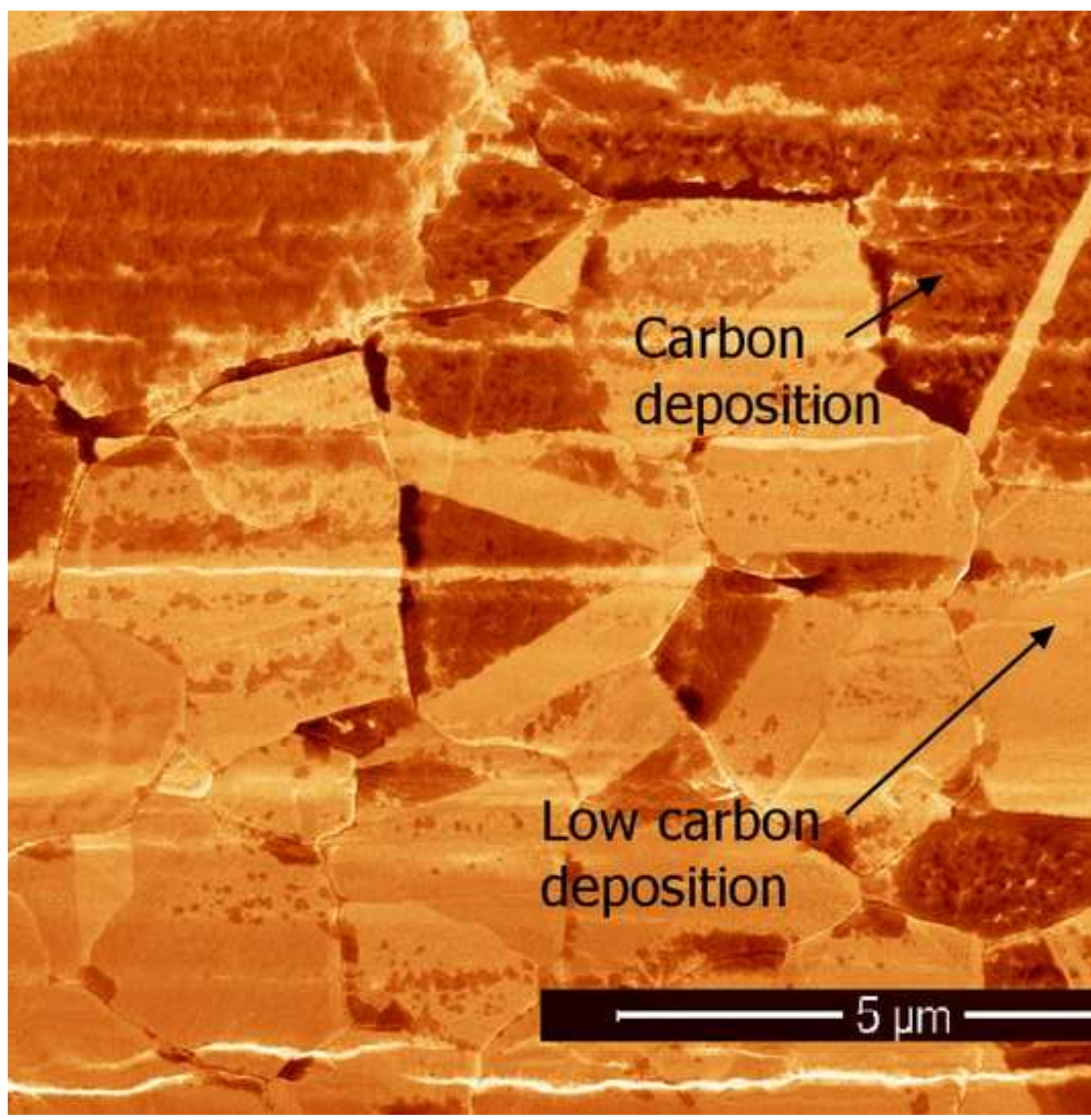


Click here to download high resolution image

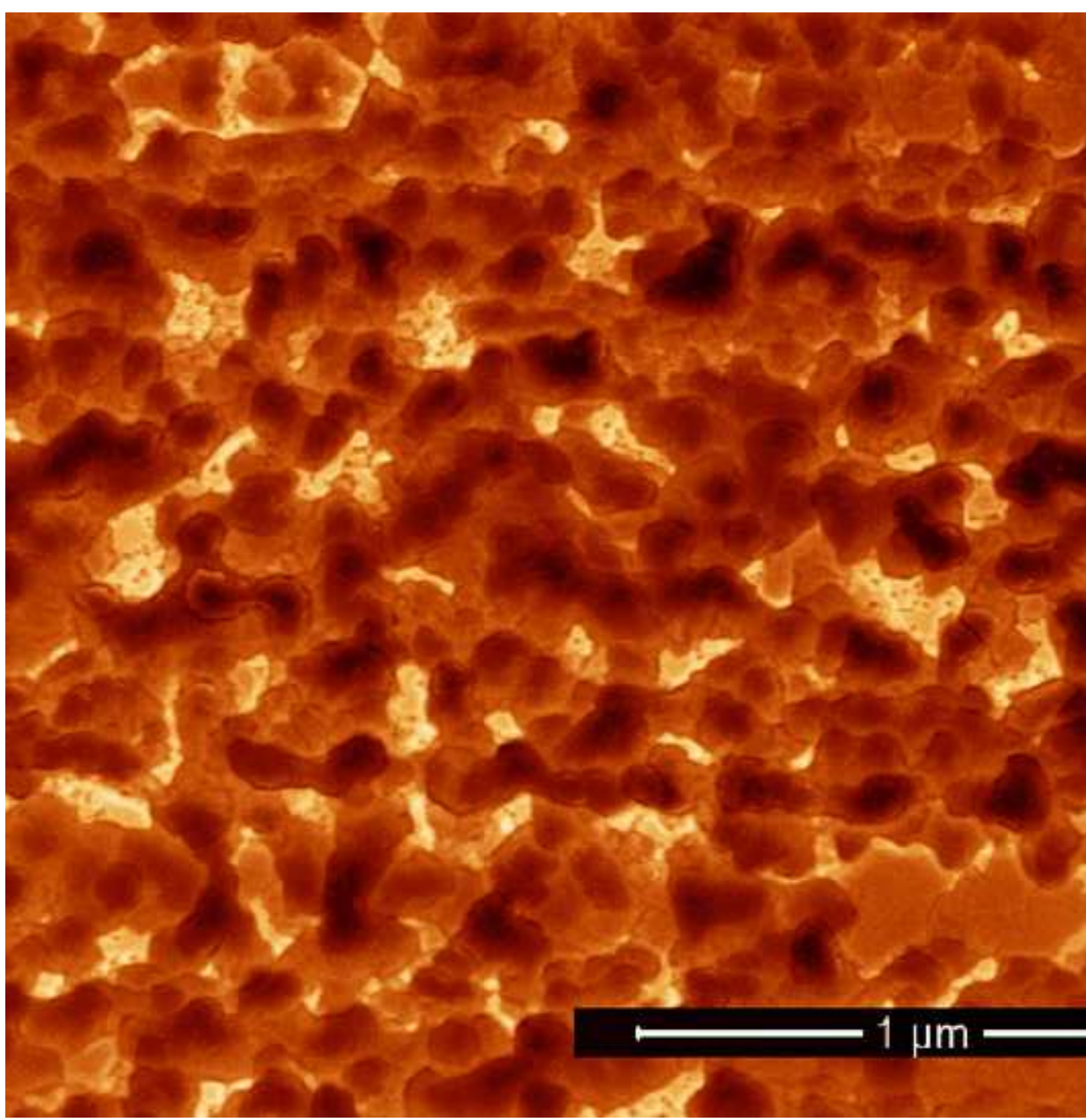




\section{Fig. 8}

Click here to download high resolution image

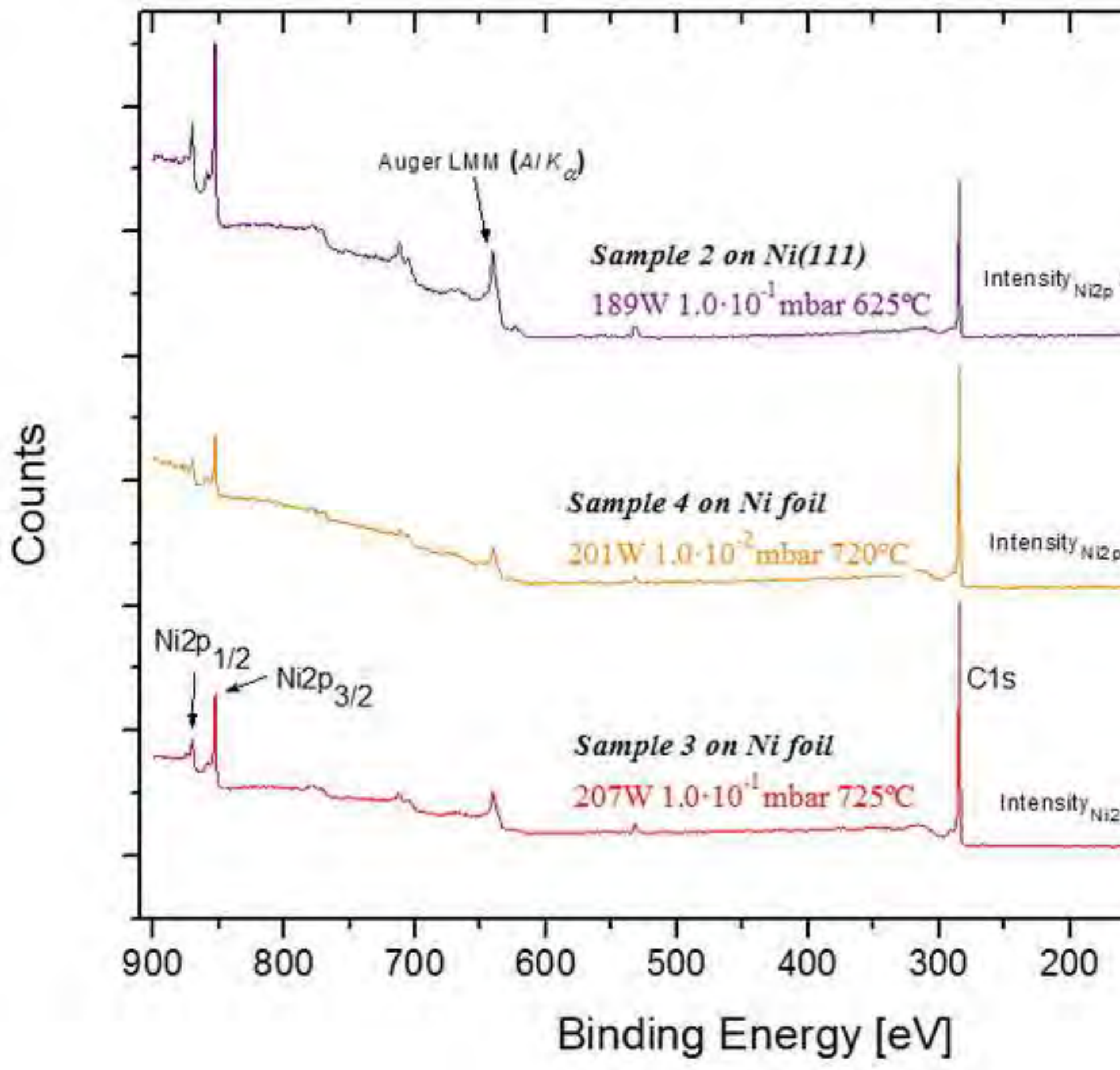


Fig. 9

Click here to download high resolution image

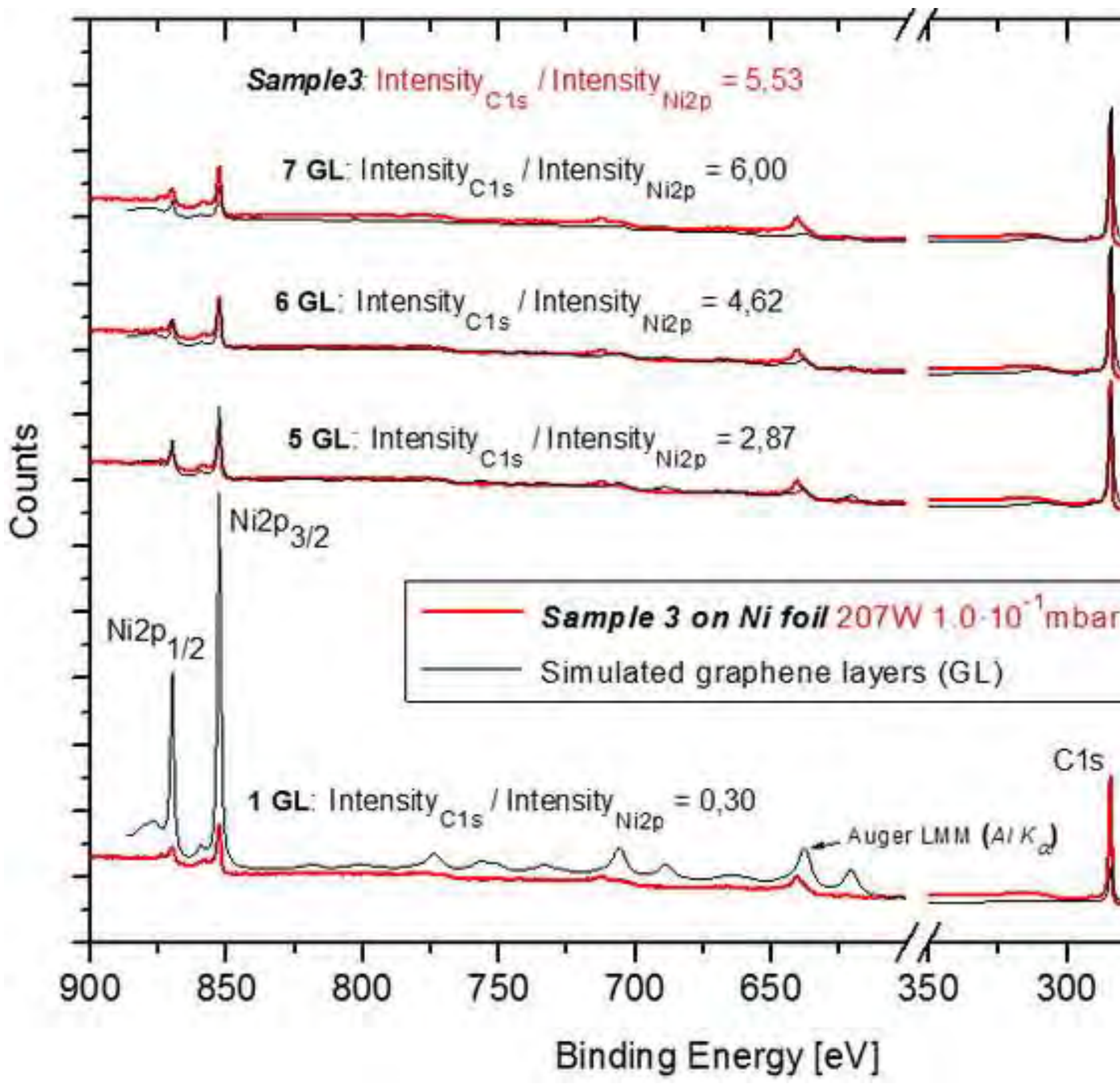


Click here to download high resolution image

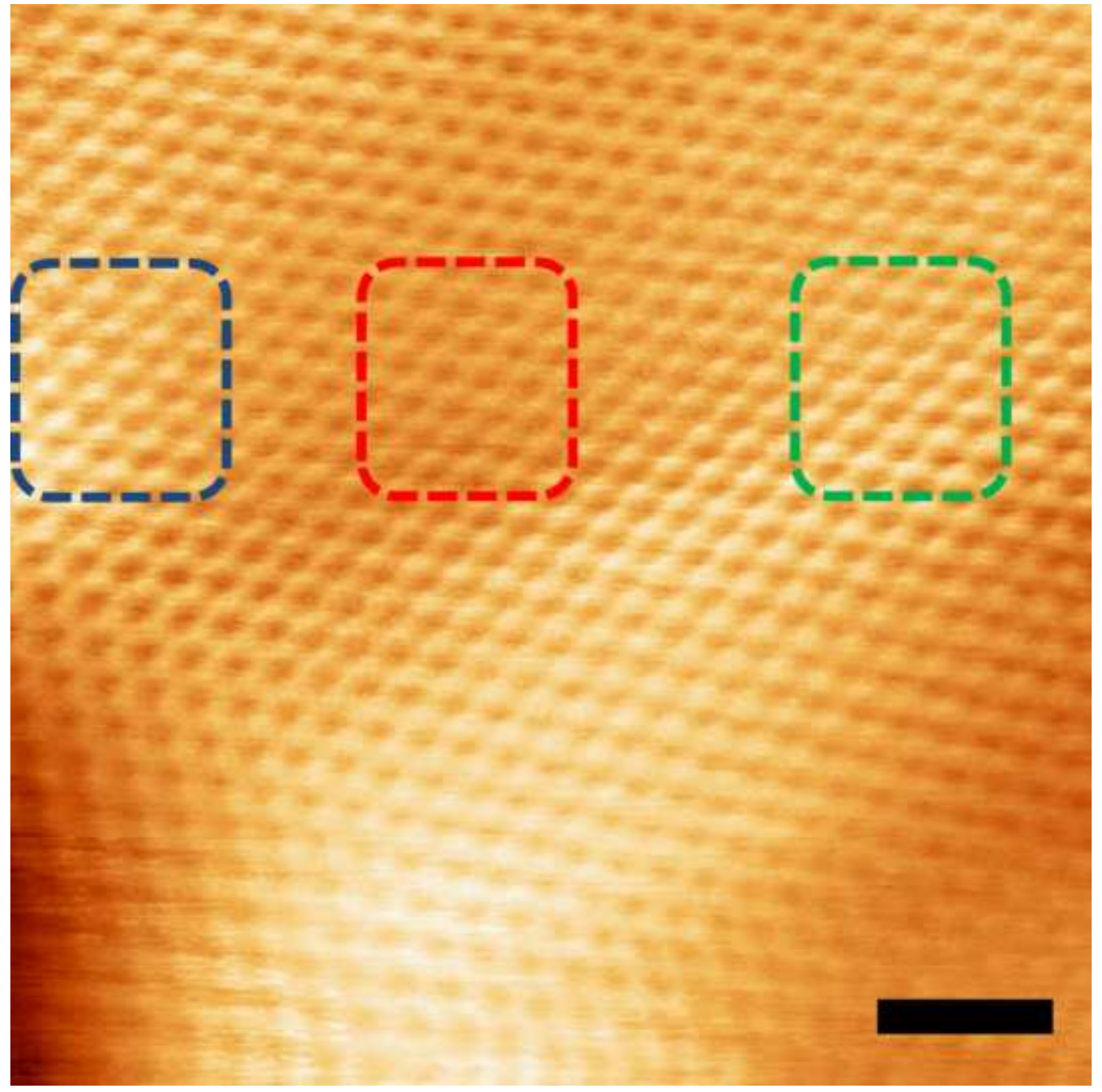


Fig. 10b

Click here to download high resolution image

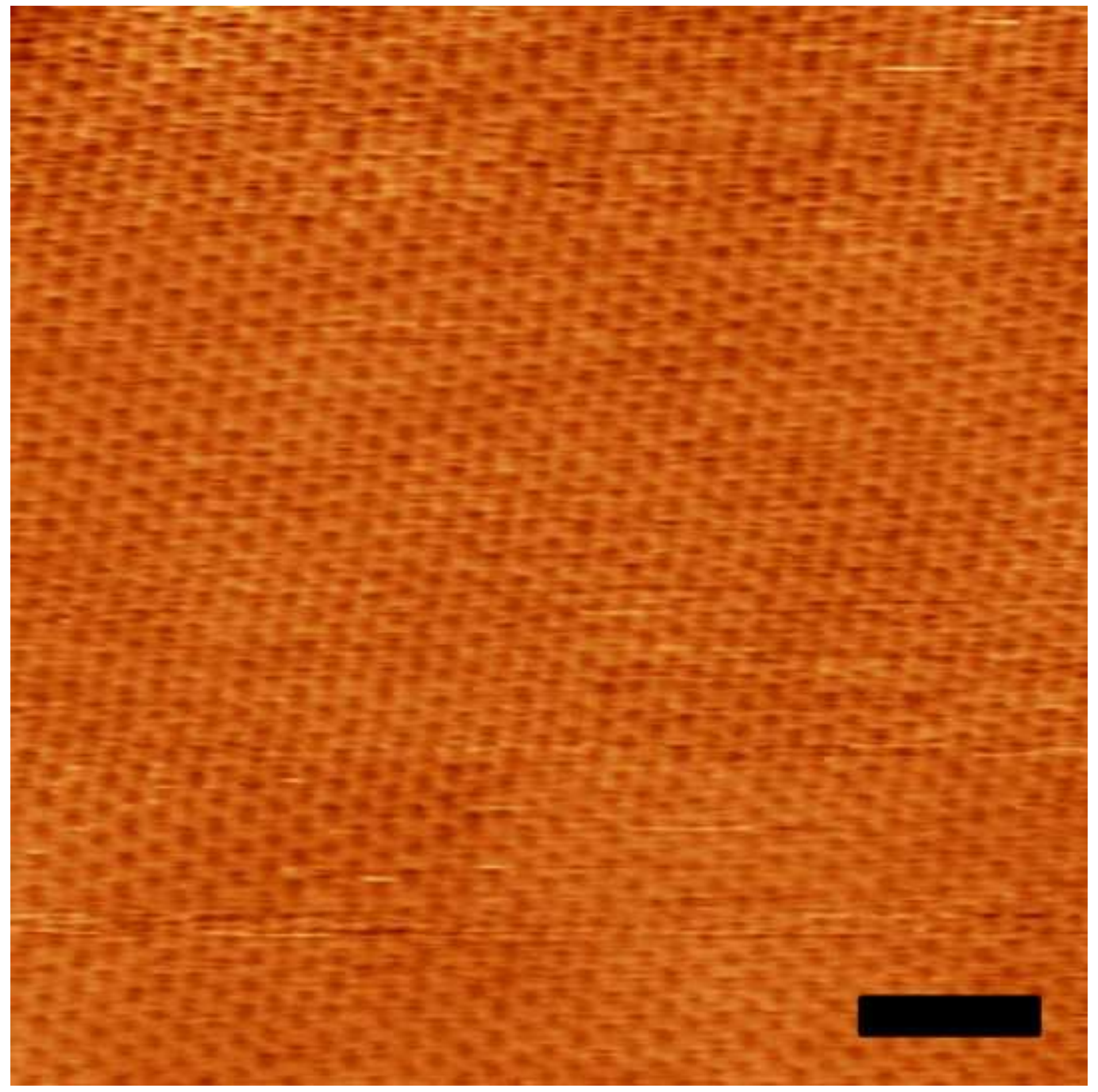


Click here to download high resolution image

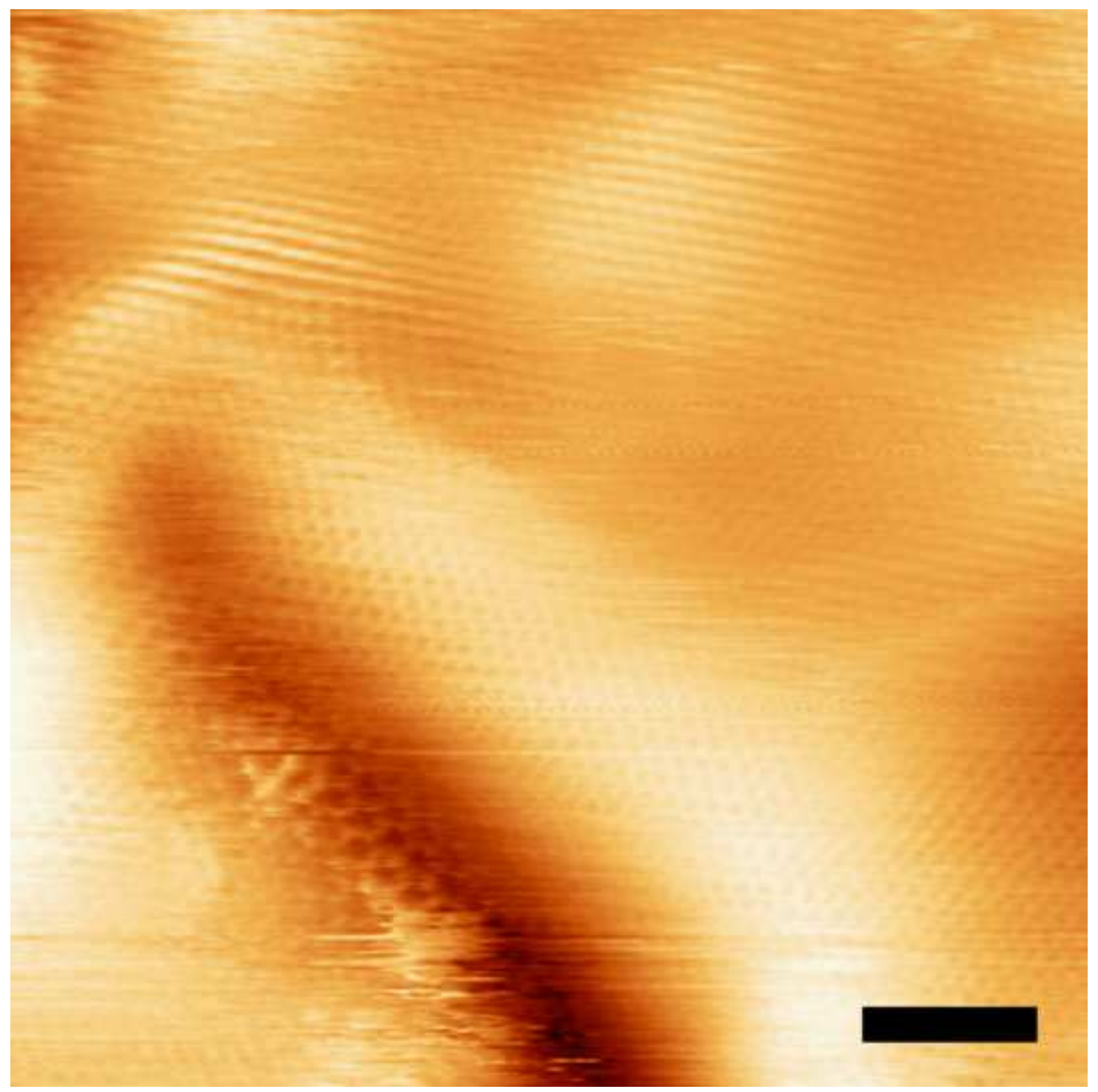


Click here to download high resolution image

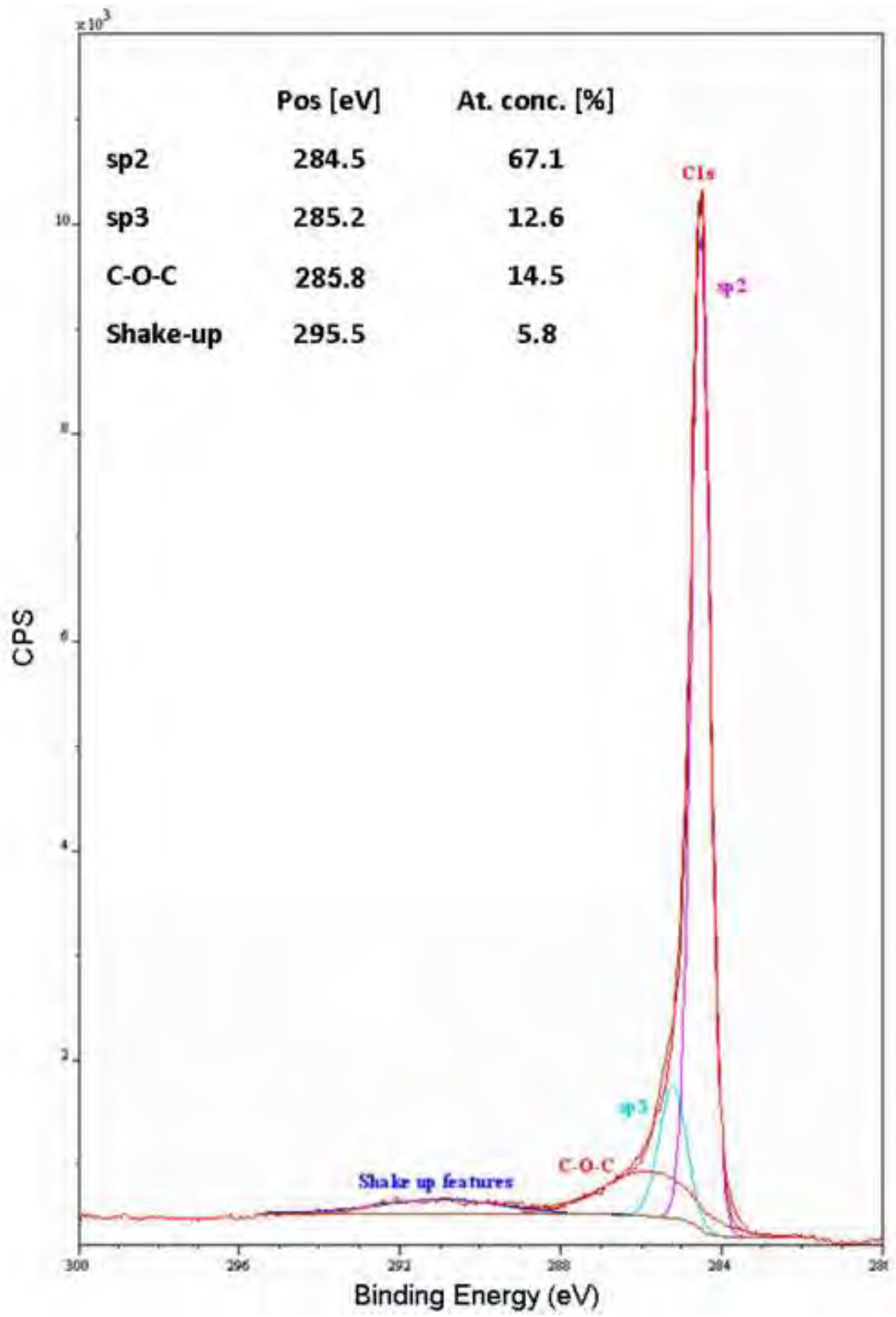


Fig. 12

Click here to download high resolution image

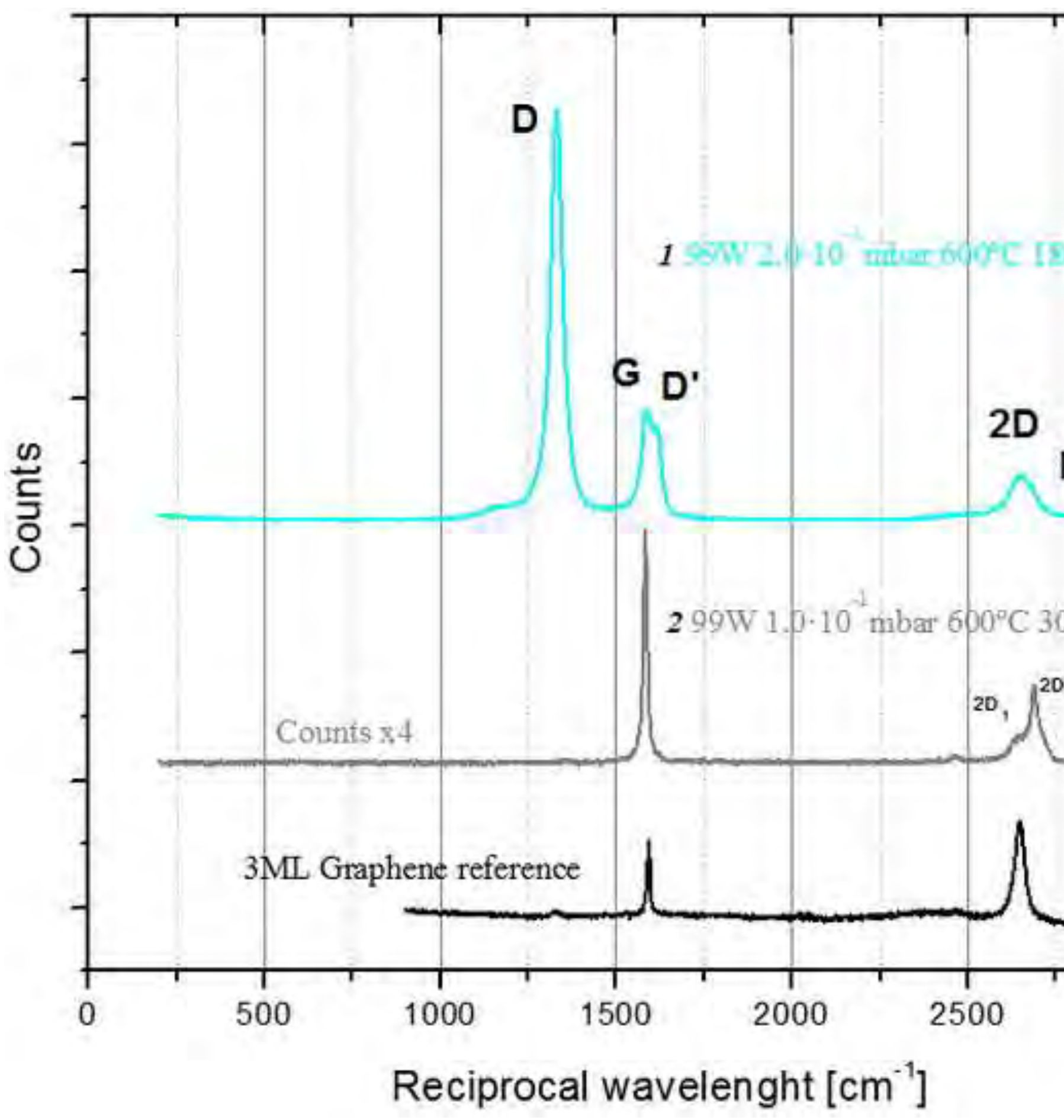


Click here to download high resolution image 
Click here to download high resolution image

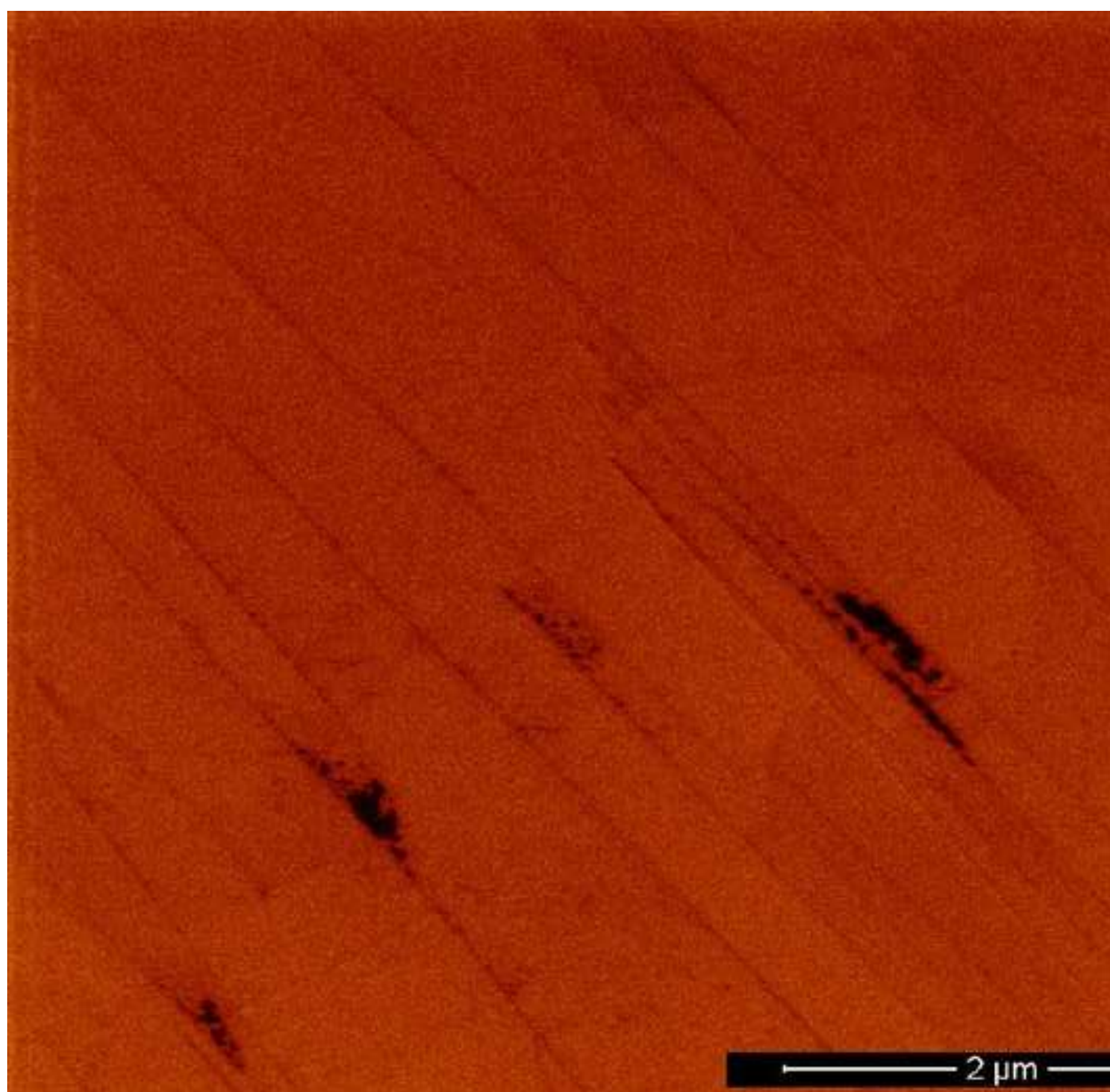




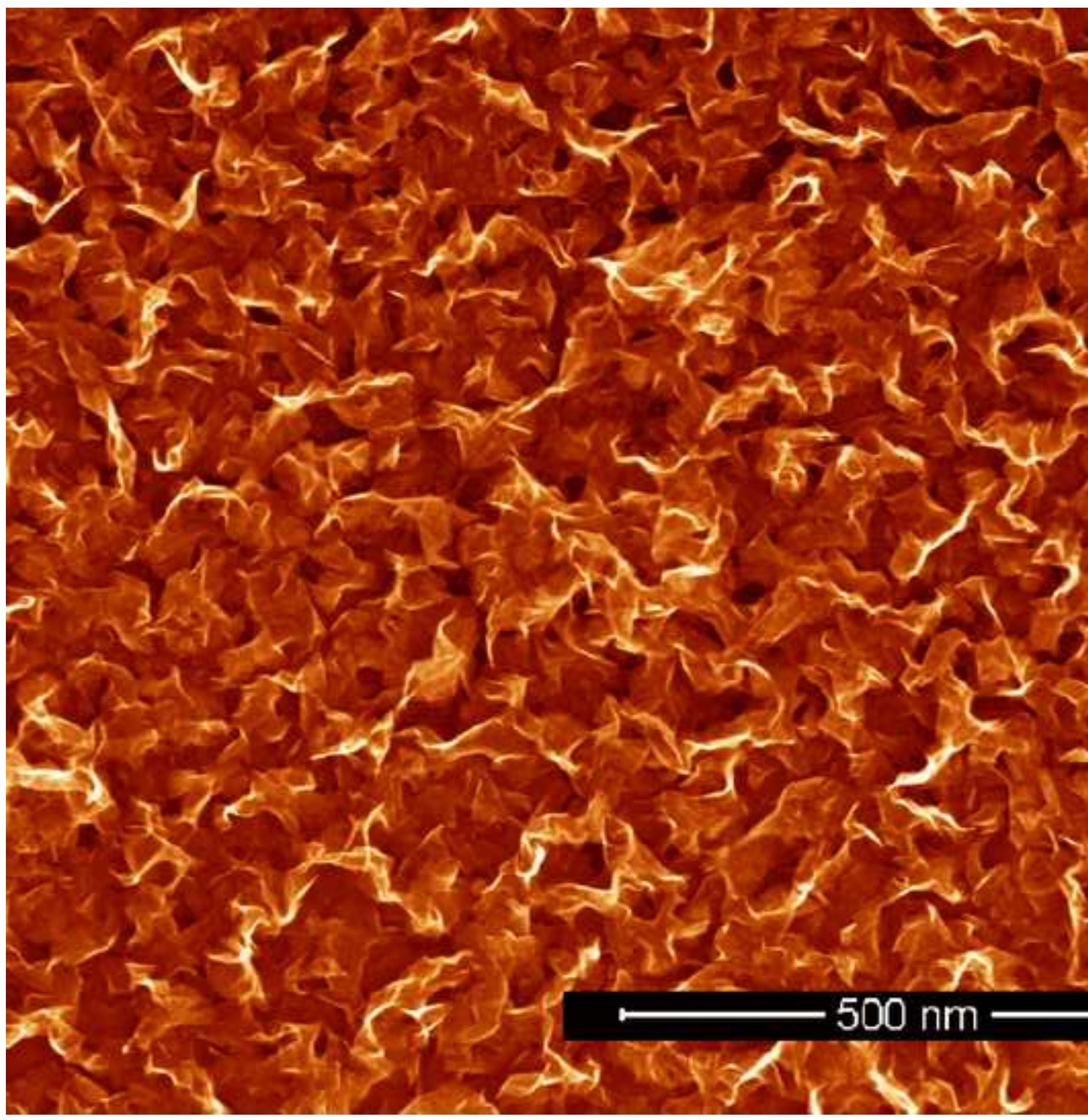


Click here to download high resolution image

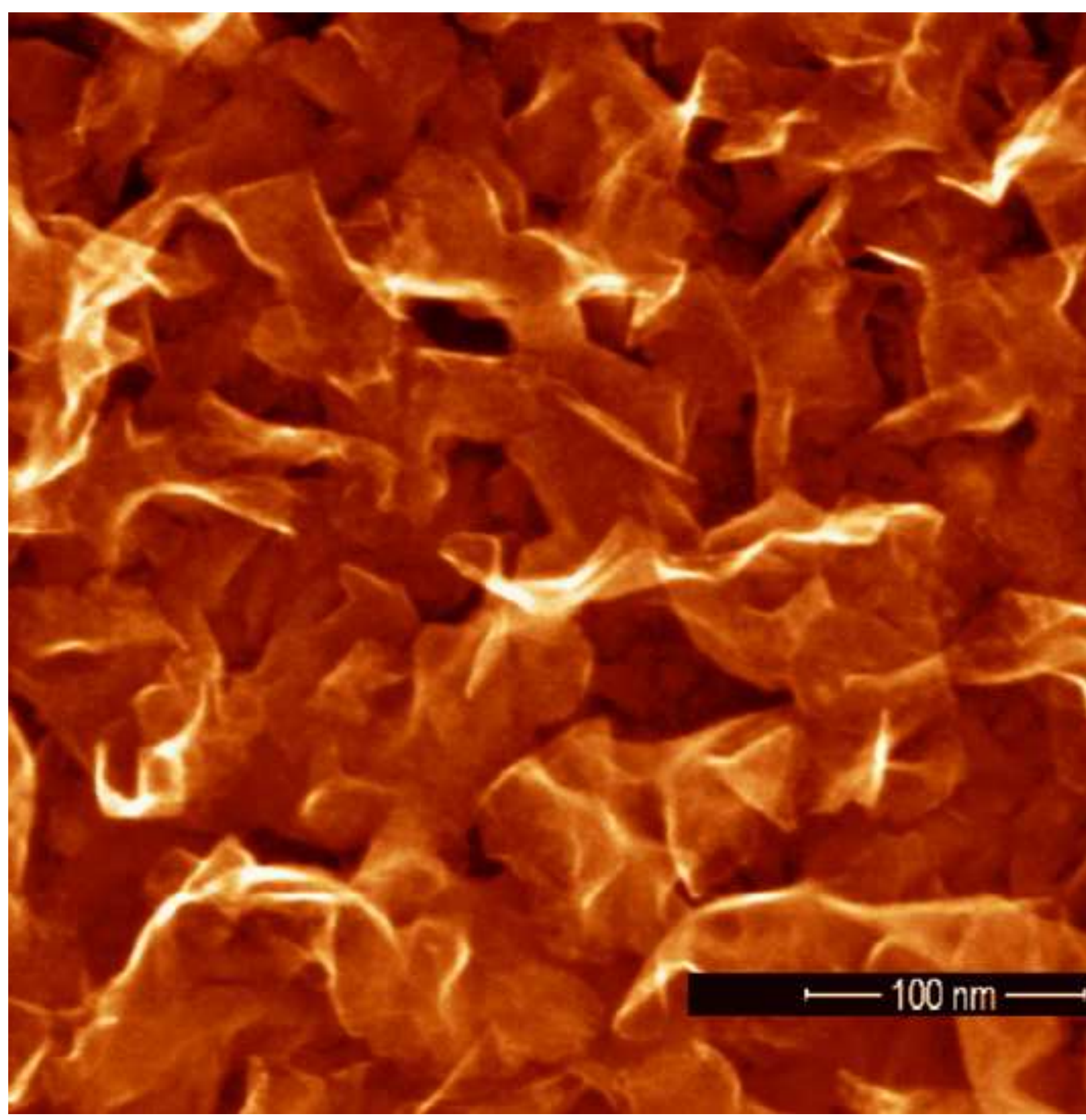


Fig. 14

Click here to download high resolution image

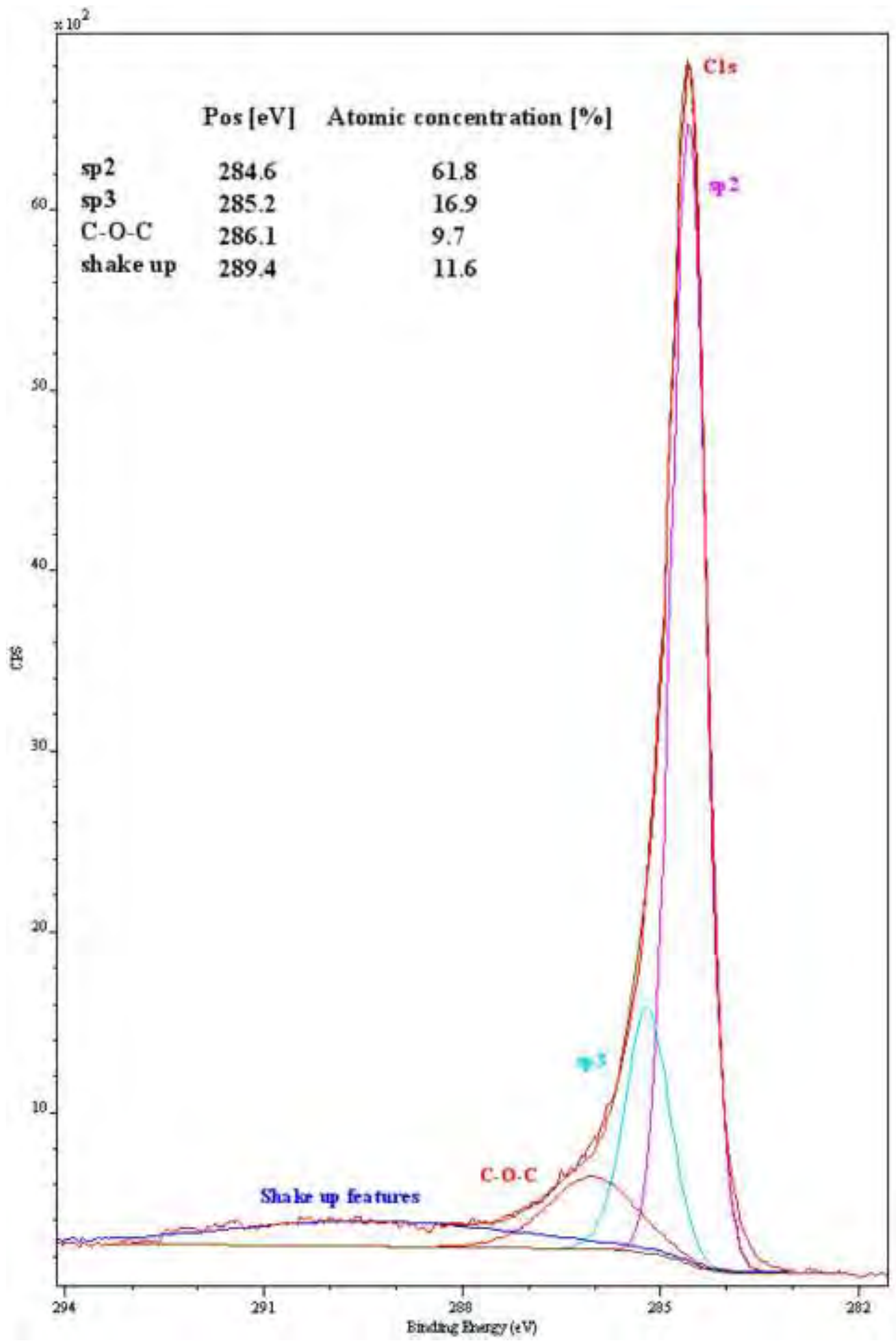

